\title{
Weighted gene coexpression network analysis-based identification of key modules and hub genes associated with drought sensitivity in rice
}

Baiyang $\mathrm{Yu}^{1+}$, Jianbin Liư ${ }^{1 \dagger}$, Di Wu${ }^{1}$, Ying Liu', Weijian Cen ${ }^{1}$, Shaokui Wang ${ }^{3}$, Rongbai $\mathrm{Li}^{1,2^{*}}$ and Jijing Luo ${ }^{1 *}$ (D)

\begin{abstract}
Background: Drought stress is an adverse factor with deleterious effects on several aspects of rice growth. However, the mechanism underlying drought resistance in rice remains unclear. To understand the molecular mechanism of the drought response in rice, drought-sensitive CSSL (Chromosome Single-substitution Segment Line) PY6 was used to map QTLs of sensitive phenotypes and to reveal the impact of the QTLs on transcriptional profiling.

Results: The QTL dss-1 was mapped onto the short arm of chromosome 1 of rice. According to transcriptomic analysis, the identified differentially expressed genes (DEGs) exhibited a downregulated pattern and were mainly enriched in photosynthesis-related GO terms, indicating that photosynthesis was greatly inhibited under drought. Further, according to weighted gene coexpression network analysis (WGCNA), specific gene modules (designating a group of genes with a similar expression pattern) were strongly correlated with $\mathrm{H}_{2} \mathrm{O}_{2}$ (4 modules) and MDA (3 modules), respectively. Likewise, $\mathrm{GO}$ analysis revealed that the photosynthesis-related $\mathrm{GO}$ terms were consistently overrepresented in $\mathrm{H}_{2} \mathrm{O}_{2}$-correlated modules. Functional annotation of the differentially expressed hub genes (DEHGs) in the $\mathrm{H}_{2} \mathrm{O}_{2}$ and MDA-correlated modules revealed cross-talk between abiotic and biotic stress responses for these genes, which were annotated as encoding WRKYs and PR family proteins, were notably differentially expressed between PY6 and PR403.
\end{abstract}

Conclusions: We speculated that drought-induced photosynthetic inhibition leads to $\mathrm{H}_{2} \mathrm{O}_{2}$ and MDA accumulation, which can then trigger the reprogramming of the rice transcriptome, including the hub genes involved in ROS scavenging, to prevent oxidative stress damage. Our results shed light on and provide deep insight into the drought resistance mechanism in rice.

Keywords: Rice, Transcriptomic profiling, Drought-sensitive phenotype, WGCNA, Photosynthesis inhibition, $\mathrm{H}_{2} \mathrm{O}_{2}$ / MDA accumulation, DEGs encoding WRKYs/PR proteins

\footnotetext{
* Correspondence: lirongbai@126.com; jjluo@gxu.edu.cn

†Baiyang Yu and Jianbin Liu contributed equally to this work.

'College of Life Science and Technology (State Key Laboratory for

Conservation and Utilization of Subtropical Agro-bioresources), Guangxi

University, Nanning 530004, China

Full list of author information is available at the end of the article
}

C The Author(s). 2020, corrected publication [2020]. Open Access This article is licensed under a Creative Commons Attribution 4.0 International License, which permits use, sharing, adaptation, distribution and reproduction in any medium or format, as long as you give appropriate credit to the original author(s) and the source, provide a link to the Creative Commons licence, and indicate if changes were made. The images or other third party material in this article are included in the article's Creative Commons licence, unless indicated otherwise in a credit line to the material. If material is not included in the article's Creative Commons licence and your intended use is not permitted by statutory regulation or exceeds the permitted use, you will need to obtain permission directly from the copyright holder. To view a copy of this licence, visit http://creativecommons.org/licenses/by/4.0/. The Creative Commons Public Domain Dedication waiver (http:// creativecommons.org/publicdomain/zero/1.0/) applies to the data made available in this article, unless otherwise stated in a credit line to the data. 


\section{Background}

Abiotic stresses such as drought, chilling, heat, and salinity are widespread factors with deleterious effects on several aspects of plants, including changes in metabolism, growth, and development, in extreme cases leading to plant death. However, as sessile organisms, higher plant usually adopt the 'overcome' strategy upon encountering any extreme environmental stresses; this strategy, in contrast to that of animals, is preferentially to opt for the tolerance or avoidance of unfavorable circumstances. In general, various tolerance/avoidance mechanisms [1], such as regulation of endogenous hormone signals [2], increases in wax deposition in the cuticle [3], and stomatal regulation [4], are employed in response to abiotic stresses.

Rice (Oryza sativa L.) is a staple food crop worldwide. Drought has been one of the major abiotic constraints of rice production with increasing frequency of global water shortages. Two important strategies, dehydration tolerance and avoidance, are employed by plants to cope with drought stress. Dehydration avoidance depends on the development of a large and deep root system to uptake water from soil and reduce water loss via the modulation of stomatal opening. Dehydration tolerance mainly enhances drought resistance via complex mechanisms that occur during later stages of drought stress, such as antioxidation processes, ABA signal transduction, osmotic adjustment, and cell membrane protection $[5,6]$. A previous reports showed that two genotypes of Persian Walnuts (Juglans regia L.), Chandler' and 'Panegine20, exhibit high osmotic stress tolerance during seed germination [7]. The further study revealed that the strong accumulation of sugars and proline were detected in radicle and plumule of drought tolerant walnut varieties during germination, suggesting that sugars and proline are involved in the osmotic adjustment of plants in response to drought stress [8].

A large number of QTLs for root growth [9], osmotic pressure regulation [10], ROS regulation, and cuticular wax accumulation, which contribute to enhanced drought resistance, have been mapped to different locations of the rice genome via map-based cloning [11-13]. DRO1, one of the genes that has been well characterized, is involved in the regulation of rice root development under drought stress. DRO1 regulates cell elongation in the root tip, which causes downward bending of the roots to avoid water deficit by increasing deep rooting [13]. DST was identified as a negative regulator of $\mathrm{H}_{2} \mathrm{O}_{2}$ accumulation in guard cells and functions in the modulation of stomatal opening. Loss of DST function increases stomatal closure, resulting in enhanced drought tolerance in rice [12]. In addition, DS8 participates in the synthesis of plant cuticular wax and in the regulation of stomatal movement in response to drought stress
[11]. Studies of these genes indicated that intricate mechanisms are employed by rice plants in the adaptation to drought stress. On the contrary, some drought stress-related QTLs were mapped through investigation of the drought sensitive traits. For example, 4 QTLs that contribute to increased transpiration rate under high vapour pressure deficit conditions were identified using $\mathrm{F}_{7}$ recombinant inbred lines of pearl millet [Pennisetum glaucum (L.) R. Br.]. All of these alleles were derived from drought-sensitive parent ICMB 841 [14]. Therefore, drought sensitivity is also an alternative trait for QTL identification in the study of drought tolerance mechanisms. In addition, an association analysis among phenotypic, genotypic, and environmental variables using single nucleotide polymorphisms (SNPs) was performed to identify the loci that are associated with drought tolerance in Persian walnut. Among candidate genes for the identified loci, majority of which are involved in ABA signaling, stomatal regulation, signal transduction, antioxidant defense, osmotic adjustment, and leaf growth and development [15].

Chloroplasts are organelles that perform photosynthesis in plant cells. As a key process for the responsive regulation, when rice plants are subjected to abiotic stresses, photosynthetic activity is greatly inhibited [16]. The inhibition of photosynthesis reduces the utilization of absorbed light energy, resulting in the generation of reactive oxygen species (ROS), including singlet oxygen, $\mathrm{H}_{2} \mathrm{O}_{2}$, and superoxide anion in chloroplasts, which in turn can cause photodamage to photosystem proteins such as D1, a core component of photosystem II [17]. Plants have evolved a series of enzymatic and nonenzymatic antioxidant systems. The enzymatic system, which involves superoxide dismutase (SOD), catalase (CAT), and ascorbate peroxidase (APX), participates in scavenging ROS and preventing damage from oxidative stress [18]. SODs function in the dismutation of $\mathrm{O}^{2-}$ to $\mathrm{H}_{2} \mathrm{O}_{2}$ and $\mathrm{O}_{2}$; the resultant $\mathrm{H}_{2} \mathrm{O}_{2}$ is then catalyzed into $\mathrm{H}_{2} \mathrm{O}$ and $\mathrm{O}_{2}$ by the activity of CAT and APX [19]. The overaccumulation of ROS, causes severe injury to plant cells, even leading to programmed cell death. Therefore, ROS homeostasis plays a key role in plant drought resistance regulation [20, 21]. It has been reported that the levels of antioxidant activity significantly enhanced with the increasing of POD, APX, CAT, SOD, and LOX enzymes in the drought tolerant genotypes of Persian walnut (Juglans regia L.) [22, 23]. Moreover, emerging evidence has indicated strong correlations among antioxidant mechanisms, photosynthesis regulation, and drought tolerance $[20,24,25]$. Specifically, ROS can act as secondary messengers that are involved in the stress signaling response, photosynthetic regulation, stomatal movement, and the development of higher plant [26]. In addition, ABA induces the accumulation of $\mathrm{H}_{2} \mathrm{O}_{2}$ via the 
activation of NADPH oxidase, which is activated by SnRK2 phosphorylation, eventually resulting in stomatal closure. However, on the other hand, the accumulation of $A B A$ induces the expression of $C A T$, suggesting that a certain relationship exists between $A B A$ signaling and $\mathrm{H}_{2} \mathrm{O}_{2}$ balance [27-32].

Recent reports have suggested that WRKY transcription factors (TFs) are involved in the regulatory mechanism pathways of abiotic stresses, including those in response to drought and salinity [33, 34]. WRKY TFs constitute a plant-specific group of zinc finger transcription factors that are characterized by containing a conserved WRKY domain and that bind to a consensus cis-element W-box (TTGACT/C) [35]. The majority of the more than 100 WRKY members in rice are involved in the plant defense response in either a negative or positive manner [35-37]. Some WRKY members have important roles in abiotic stress responses; for example, OsWRKY13 has been suggested to regulate the antagonistic cross-talk between drought and disease resistance pathways via repression of SNAC1 and WRKY45-1 [33, 34]. Moreover, pathogenesisrelated (PR) proteins have been well characterized as a group of proteins that are induced not only by infection from pathogens, such as viruses, bacteria, and fungi, but also in response to abiotic stresses, including drought and salinity [38]. All PR proteins in both monocots and dicotyledons can be classified into 17 families [39]. PR3 and PR4 proteins have plant chitinase activity, and the PR5 family of proteins includes permatins, osmotins, zeanatins, and thaumatin-like proteins [40]. PR10 genes belong to a multigene family in various plant species and exert RNase activity and ligand binding activity and are involved in posttranslational modification (phosphorylation) and phytohormone signaling in response to stress. The promoters of PR10 genes harbor cis-acting elements for WRKY, bZIP, ERF, and MYB transcription factors, suggesting PR10 genes have pivotal roles in the stress response pathways [40].

Given the rapid development of next-generation sequencing technology, RNA-seq has been widely used to explore the transcriptomic profiling of plants in response to challenges with adverse environmental conditions. Recent studies have revealed that antioxidant processes, the ABA pathway, and photosynthesis are the target biological processes for plants in response to environmental changes at the transcription level [41, 42]. Intriguingly, the photosynthesis rate was found to decline under drought stress, especially in drought-sensitive genotypes, suggesting that the enhancement of antioxidant capacity favors maintaining a higher photosynthetic activity and thereby improves the drought resistance of plants [42]. Moreover, phytohormone signaling and $\mathrm{Ca}^{2+}$ signaling have also been highlighted in transcript profiling and have been revealed cross-talk among phytohormone signaling pathways $[43,44]$. In addition, a large number of transcription factor families, such as WRKY, bZIP, and MYB families, were found to be involved in ABAmediated signal transduction under abiotic stress $[45,46]$. However, the molecular mechanism of drought tolerance/ sensitivity still needs to be further investigated.

Weighted gene coexpression network analysis (WGCN A) is a new technique that can be used to identify potential gene modules with the highest connectivity among genes, and these modules are correlated with certain phenotypes among the gene expression data [47, 48]. WGCNA involves the construction of a coexpression network that can indicate correlations among genes across samples. Based on the coexpression relationships, genes with similar expression are grouped into the same module, thus suggesting that genes in the same module may have similar functions or possibly have common biological regulatory roles [49]. This method has been successfully applied in numerous study cases that have been used to identify hub genes and to determine the relationships between gene expression data and relevant phenotypes [50-55].

Here, we identified a drought-sensitive rice CSSL PY6, which carries the substitution segment derived from the drought-sensitive variety Lambayeque 1 , by backcrossing into the background of the drought-tolerant variety PR403. The $d s s-1$ locus underlying the drought-sensitive phenotype of PY6 was harbored on the short arm of chromosome 1 . To investigate the impact of $d s s-1$ on the transcriptional profiling of PY6, RNA-seq was performed to identify genes that were differentially expressed under drought stress treatment. Further, WGCNA was applied to identify hub genes from the modules that were strongly correlated with droughtinduced physiological indicators. By using this approach, we aimed to elucidate the $d s s$-1-mediated potential regulatory network that is related to drought stress tolerance in rice.

\section{Results \\ Identification of the dss-1 locus for drought-sensitive phenotypes in CSSLs}

A set of 140 rice CSSLs was constructed by introgressing the genomic segments of the drought-sensitive variety Lambayeque 1 into the genome of the drought-resistant variety PR403 through backcrossing (unpublished data; Fig. 1a). To elucidate the molecular regulatory mechanism underlying the drought-sensitive phenotype of Lambayeque 1, a drought stress treatment was applied to identify the major QTLs for drought-sensitive traits. PY6, one of the CSSLs, was highlighted for its droughtsensitive phenotype. After 10 days of drought treatment, the treated CSSLs were rewatered and allowed to recover for 3 days. In the screening, PY6, a CSSL, was 


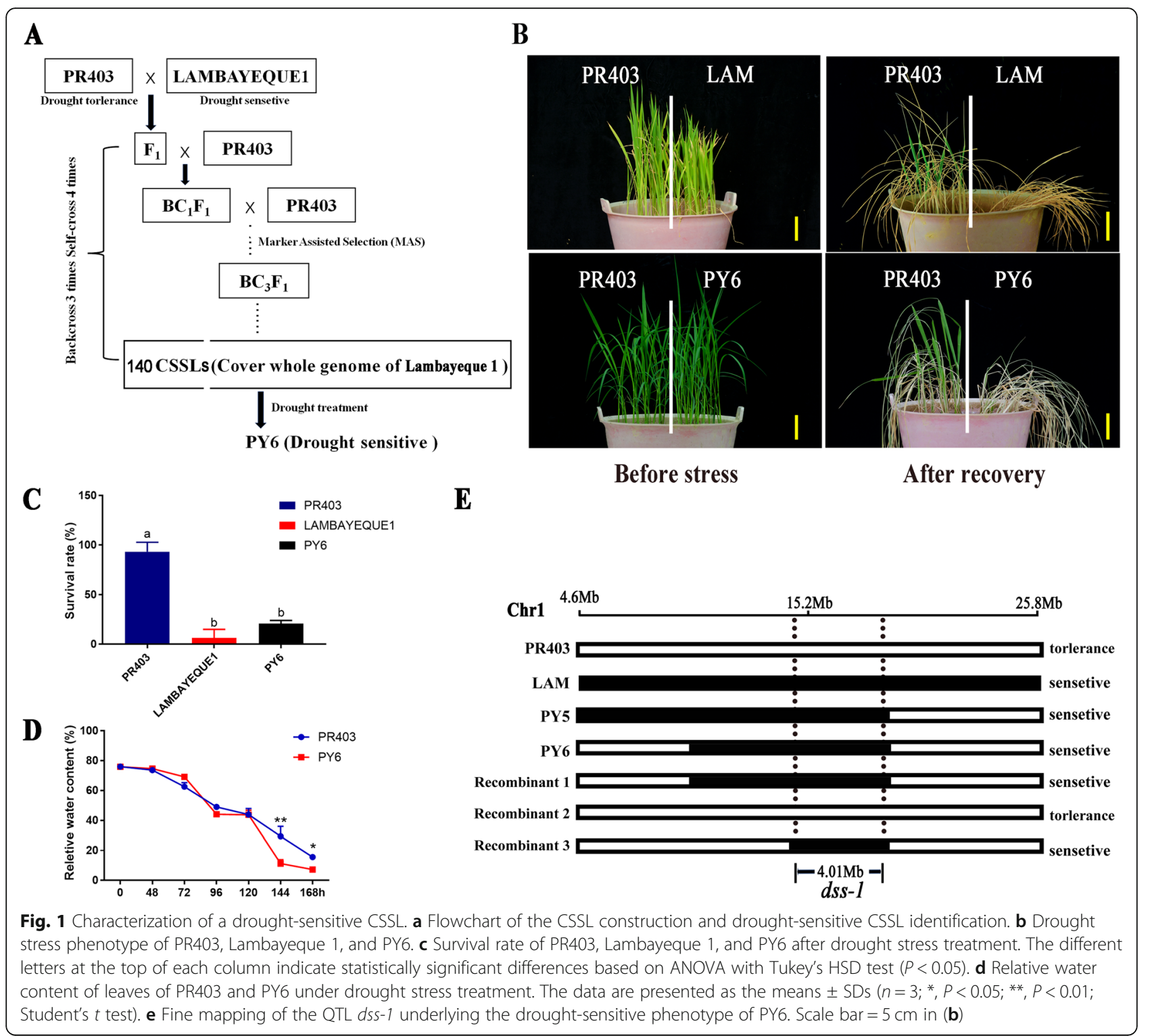

identified as presenting a significant difference in survival rate $(20.0 \%)$ compared with its recurrent parent PR403 (93.3\%), whereas no significant difference was observed in the survival rate of PY6 and its donor parent Lambayeque 1 (Fig. 1b-c). During the drought stress treatment, the relative water content of the seedlings declined in PY6 and PR403 with decreasing soil moisture. However, starting at $120 \mathrm{~h}$, a significant difference was observed between PY6 and PR403, and the former exhibited more severe dehydration at the later stage of the treatment, suggesting that drought stress disrupted the balance of water uptake and evaporation in PY6 (Fig. 1d). PY6 carries a 10-Mb chromosome segment derived from Lambayeque 1 on the short arm of chromosome 1. These results suggested that the segment from Lambayeque 1 may harbor a major QTL locus for the drought-sensitive phenotype of PY6. We designated this QTL as dss-1.

To reveal the genetic basis underlying the droughtsensitive phenotype afforded by the $d s s-1$ locus, PY6 was backcrossed with the recurrent parent PR403 to construct a mapping population. The $F_{1}$ plants exhibited drought resistance, which was similar to that of the parental line PR403. Then, an $F_{2}$ population was generated to determine the inherited features of $d s s-1$. In a 151individual $F_{2}$ population, 111 members showed drought resistance, and 40 showed drought-sensitive traits (resistant:sensitive $\approx 3: 1, \chi^{2}=0.1788<\chi^{2} \quad 0.05=3.84$ ), indicating that drought-sensitive trait is controlled by a single recessive gene. In turn, the recombinants were screened for genetic linkage analysis, and $d s s-1$ was ultimately narrowed down to a 4.01-Mb interval (Fig. 1e). 


\section{Drought induced $\mathrm{H}_{2} \mathrm{O}_{2}$ and MDA accumulation in the leaves of PY6}

Given that previous reports indicated that drought stress was known to inhibit photosynthetic activity in plants due to an imbalance between light capture and its utilization [56] and coupled with the considerable potential for increased accumulation of superoxide and hydrogen peroxide in chloroplasts [57], we examined the accumulation of ROS and the activity of its scavenging enzymes in the leaves of drought-treated PY6 plants. Leaf samples were harvested at 4 sampling points (Fig. S1). In contrast to PR403, the increased accumulation of $\mathrm{H}_{2} \mathrm{O}_{2}$ was detected in PY6 from points 40 to 15 , whereas the enhanced content could be observed only at point 22 in PR403; therefore, the significantly increased accumulation of $\mathrm{H}_{2} \mathrm{O}_{2}$ was detected in PY6 at sampling points 22 and 15 compared with that of their respective controls (Fig. 2a). The marked accumulation of $\mathrm{H}_{2} \mathrm{O}_{2}$ in the leaves of PY6 was confirmed by $\mathrm{DAB}$ staining (Fig. 2b). Consistently, the activity of POD, an enzyme for $\mathrm{H}_{2} \mathrm{O}_{2}$ scavenging, was upregulated in response to the accumulation of $\mathrm{H}_{2} \mathrm{O}_{2}$ (Fig. 2c). However, the activity of APX and CAT, another two major enzymes for $\mathrm{H}_{2} \mathrm{O}_{2}$ scavenging, was not induced, and no significant difference was observed between either genotype during drought treatment (Fig. S2A, B). For the dynamic changes in superoxide activity, the content of SOA (superoxide anion) and the activity of its scavenging enzyme SOD at point 15 exhibited significant differences between the two genotypes with higher values in PY6. However, the content of SOA in the whole period of drought treatment was not significantly greater than that in the untreated control (point 40) (Fig. 2d, e; Fig. S2C). Moreover, the contents of MDA, a major reactive aldehyde resulting from the peroxidation of polyunsaturated fatty acid (PUFA) constituents of biological membranes and a compound that acts as an indicator of abiotic stress-induced biomembrane damage, were significantly higher in PY6 than in PR403 at points 22 and 15 (Fig. 2f).

Taken together, our results suggest that the droughtsensitive phenotype may be caused by the overaccumulation of ROS, especially $\mathrm{H}_{2} \mathrm{O}_{2}$, which could result in severe damage to plant cells and peroxidation of the cell membrane, thereby leading to the dehydration of rice plants under drought stress.

\section{Accumulation of $\mathrm{ABA}$ and stomatal modulation during drought stress treatment}

As mentioned above, PY6 exhibited more severe dehydration at the later stage of treatment compared with the early stage, suggesting that PY6 either had a higher transpiration rate or lower water uptake ability when subjected to drought stress. ABA is a key regulator of plant stress responses and regulates a series of physiological processes, including tolerance to abiotic stresses and stomatal closure [58]. To determine the role of ABA-mediated stomatal movement in drought-induced severe dehydration in PY6, the ABA content and stomatal aperture status in droughttreated samples were examined. ABA accumulated during drought stress treatment, although no significant difference between PY6 and PR403 was observed at any sampling point (Fig. S2D). Scanning electron microscopy was used to examine the stomata status of leaf samples. Compared with that in the untreated control samples, the percentage of completely closed stomata in the droughttreated samples was significantly greater. However, PY6 and PR403 exhibited similar patterns in the ability to modulate their stomata in response to drought stress, and no significant difference in stomatal density was observed for either genotype (Fig. S2E). Therefore, these results suggested that severe dehydration in drought-treated PY6 do

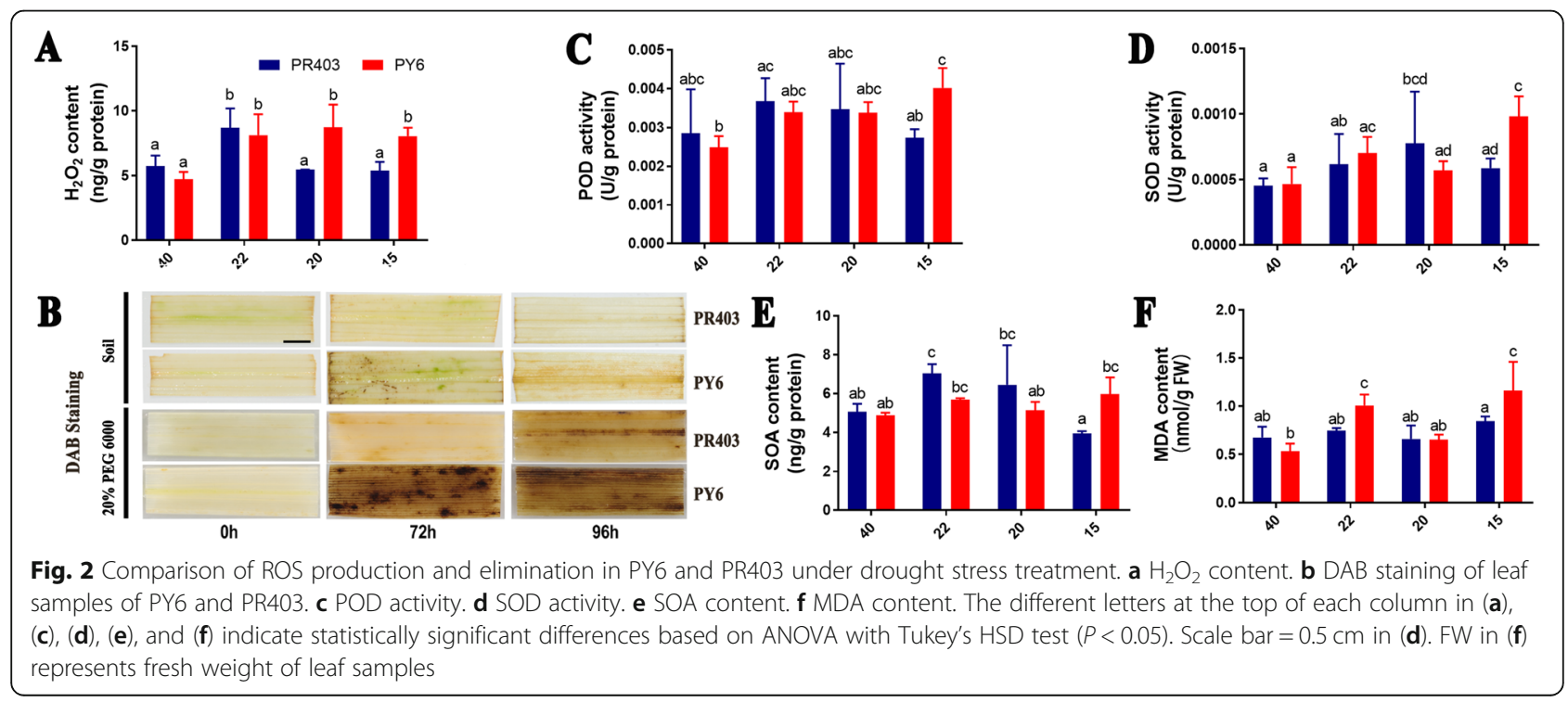


not result from a disruption of ABA-mediated stomatal transpiration regulation but, rather, may be caused by the limitation of water uptake or by other water loss pathways.

\section{Comparative transcriptional profiling of PY6 under drought stress}

To further reveal the impact of $d s s-1$ on drought stressinduced $\mathrm{H}_{2} \mathrm{O}_{2}$ accumulation that may lead to the drought sensitivity of PY6, we investigated the transcriptional profiling of PY6 and PR403 in response to drought stress through RNA-seq. Principal component analysis (PCA) was initially performed based on all the transcriptional profiling data to detect the variations among the samples under the drought treatment (Fig. 3a). PC1 explained $37.98 \%$ of the total variation. The samples of CK40 and CK22/20 of both PY6 and PR403 were clearly separated by PC1, indicating the effect of the development imposed on the variation, while PC1 also separated the drought-treated samples of point 22 from those of point 20, indicating an effect of drought treatment on the transcriptomes of the samples. PC2 explained $27.94 \%$ of the total variation and clearly separated drought-treated samples from the parallel untreated samples (Fig. 3a). Moreover, both PY6 and PR403 samples from point 20 exhibited marked divergence from other sampling points, indicating that dramatic transcriptomic reprogramming occurred at this sampling point.

Drought-induced differentially expressed genes (DEGs) were identified by comparison with untreated controls (Table S2). It is worth noting that the developmentdependent DEGs were detected in the same sampling points of well-watered samples under the same criteria, and they were excluded from the DEG set to avoid misinterpretation of drought-induced transcriptomic responses (Table S2-1 -8). Finally, at the two sampling points of PR403, 3052 DEGs, including 1135 upregulated and 1917 downregulated DEGs, were detected at point 22 (Table S2-9), while 5821 DEGs were detected at point 20, including 2383 upregulated and 3438 downregulated DEGs (Table S2-10). In PY6, 3145 (1748 upregulated/1397 downregulated) and 5529 (2655 upregulated/ 2874 downregulated) DEGs were detected at points 22 and 20, respectively (Table 1 ; Table S2-11, - 12). Among the resultant DEGs, Venn diagram analysis

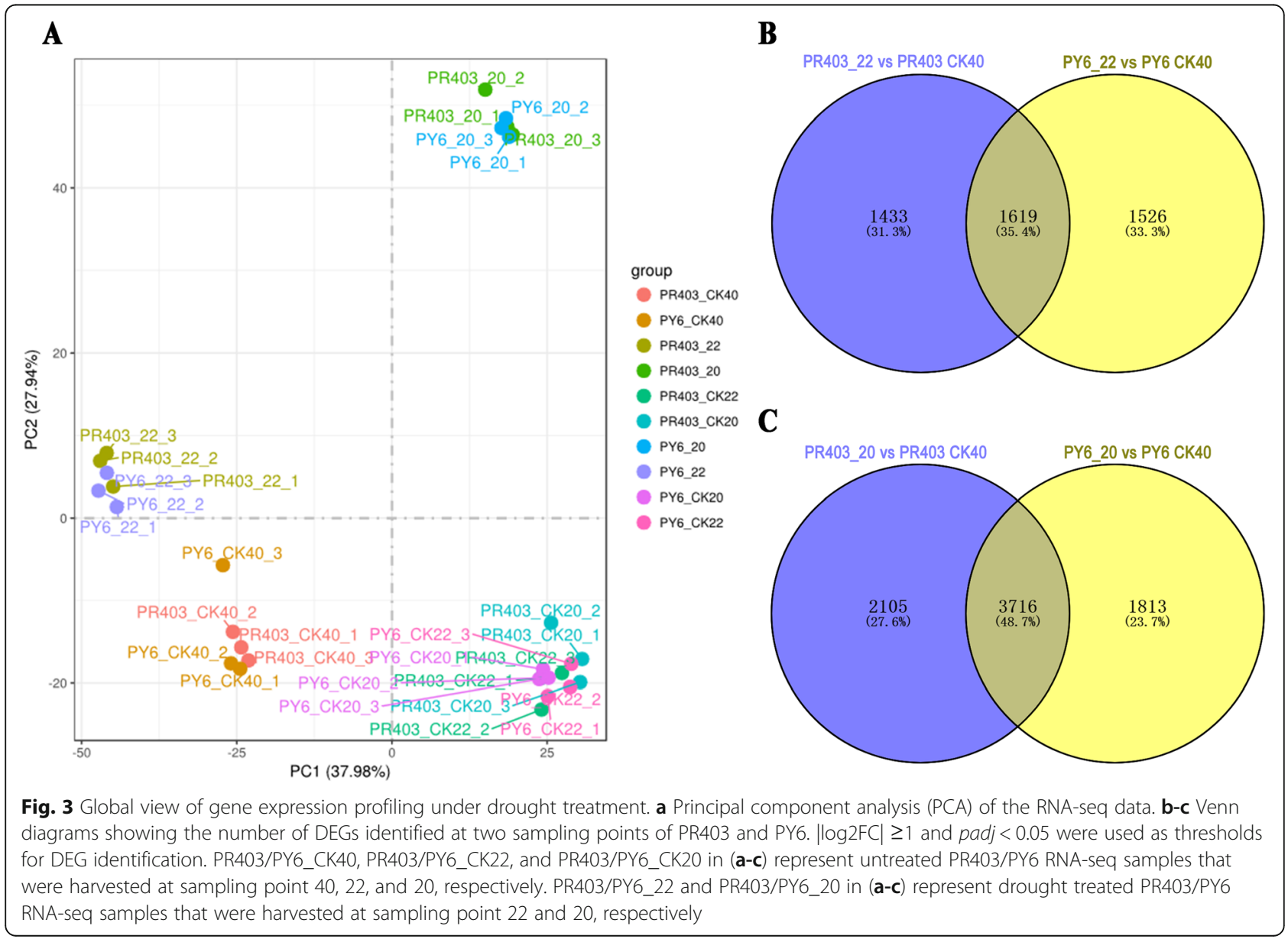


Table 1 DEGs detected in PR403 and PY6 under drought stress treatment

\begin{tabular}{llll}
\hline Genotypes & Category of DEG & \multicolumn{2}{l}{ Sampling point } \\
\cline { 3 - 4 } & & $\mathbf{2 2}$ & $\mathbf{2 0}$ \\
\hline PR403 & Up-regulated & 1135 & 2383 \\
& Down-regulated & 1917 & 3438 \\
\multirow{3}{*}{ PY6 } & Total & 3052 & 5821 \\
& Up-regulated & 1748 & 2655 \\
& Down-regulated & 1397 & 2874 \\
\multirow{4}{*}{ Common } & Total & 3145 & 5529 \\
& Up-regulated & 459 & 1202 \\
& Down-regulated & 683 & 1982 \\
& Opposite-regulated & 477 & 532 \\
& Total & 1619 & 3716 \\
\hline
\end{tabular}

revealed that 1142 DEGs were common between PR403 and PY6 at point 22, while 3184 were common between PR403 and PY6 at point 20, which accounted for 38.4 and $48.7 \%$ of the total DEGs at the two sampling points, respectively (Fig. 3b-c; Table 1 ). These findings demonstrated that PY6 has a similar genetic background and a drought stress response as PR403.

To verify the RNA-seq data quality, five genes were randomly selected for qRT-PCR. Similar trends of expression changes were observed between the qRT-PCR and RNAseq data, indicating that the transcriptomic data were reliable for further analysis (Fig. S3A-E; Table S1).

\section{Gene ontology enrichment analysis}

GO enrichment analysis was performed to cluster the identified DEGs into the functional categories of biological process (BP), cellular component (CC), and molecular function (MF) subgroups. The results showed that all the DEGs were enriched in the GO terms that were related to photosynthesis in the chloroplasts (Fig. 4a-d, $P \leq 0.01$ ). For instance, in terms of the BP group, the GO terms involving photosynthesis (GO: 0015979); photosynthesis, light harvesting (GO: 0009765); photosynthesis, light harvesting in photosystem I (GO:0009768); photosynthesis, light reaction (GO:0019684); chlorophyll biosynthetic process (GO: 0015995); and porphyrin-containing compound biosynthetic process (GO:0006779) were highly significantly overrepresented. The overrepresented GO terms, such as those involving chloroplast part (GO:0044434), the chloroplast envelope (GO:0009941), chloroplast thylakoid (GO:0009534), the chloroplast thylakoid membrane (GO:0009535), and the chloroplast thylakoid lumen (GO:0009543) in the CC category (Fig. S4A-D, $P \leq 0.01$ ), and the overrepresented terms of the MF category, such as pigment binding (GO:0031409) and chlorophyll binding (GO:0016168) (Fig. S5A-D, $P \leq 0.01$ ), supported the identified DEGs are related to photosynthetic modulation in response to drought stress. In addition, the superoxide metabolism-related GO terms superoxide metabolic process (GO:0006801) and regulation of superoxide metabolic process (GO:0090322) were highly enriched among the DEGs identified at point 20 of PR403, suggesting the involvement of ROS scavenging processes in the drought stress response (Fig. 4c, $P \leq$ 0.01 ). GO enrichment analysis suggested that photosynthetic modulation plays an important role in the regulatory network of rice plants in response to drought stress; therefore, chloroplasts are target organelles for the drought stress response.

\section{Construction of the coexpression network and identification of drought-induced hub genes}

To further investigate the impact of $d s s-1$, a major QTL conferred PY6 drought sensitive phenotype, on the regulatory network in response to drought stress and to identify the specific genes that are strongly correlated with drought-induced physiological alterations in rice, we performed a WGCNA. After removing the genes with a low FPKM $($ FPKM $<1)$, a total of 23,178 genes were used to construct a scale-free coexpression network based on the soft-thresholding power of $\beta=12$ (Fig. S6). According to the WGCNA results, the clusters with highly interconnected genes were defined as modules, and the genes in the same modules had high correlation coefficients. A total of 26 modules (coded with different colors to indicate different modules) were identified via the Dynamic Tree Cut method (core parameter: MEDissThres $=0.25)$ (Fig. 5a). With respect to the correlations of physiological traits with overrepresented modules, intriguing results were observed in the correlations. In general, the modules black, blue, and tan were negatively correlated with the majority of physiological traits, whereas the modules royalblue, brown, red, grey60, orange, and green were positively correlated with the traits, indicating that the genes clustered in a module have similar altered expression patterns in response to drought stress (Fig. 5b). Considering that significant differences in the contents of $\mathrm{H}_{2} \mathrm{O}_{2}$ and MDA were observed during drought stress, we focused on the correlations of $\mathrm{H}_{2} \mathrm{O}_{2}$ and MDA with gene modules and identified hub genes that were strongly correlated with the two drought-related physiological indicators (Fig. 2a, b, f; Fig. 5b, c, d). With the cut-off threshold of GS (Gene Significance) $>0.4$, in all 26 of the modules, black (741 genes; $R^{2}=-0.69, P=3.0 \times 10^{-5}$ ), blue (4810 genes; $\left.\mathrm{R}^{2}=-0.57, P=9.0 \times 10^{-4}\right)$, grey60 (148 genes; $\mathrm{R}^{2}=0.67$, $\left.P=4.0 \times 10^{-5}\right)$, and green (1099 genes; $R^{2}=0.61, P=$ $3.0 \times 10^{-4}$ ) were considered the modules that were strongly correlated with $\mathrm{H}_{2} \mathrm{O}_{2}$, and red (913 genes; $\mathrm{R}^{2}=$ $\left.0.63, P=2.0 \times 10^{-4}\right)$, brown (3132 genes; $\mathrm{R}^{2}=0.56, P=$ 


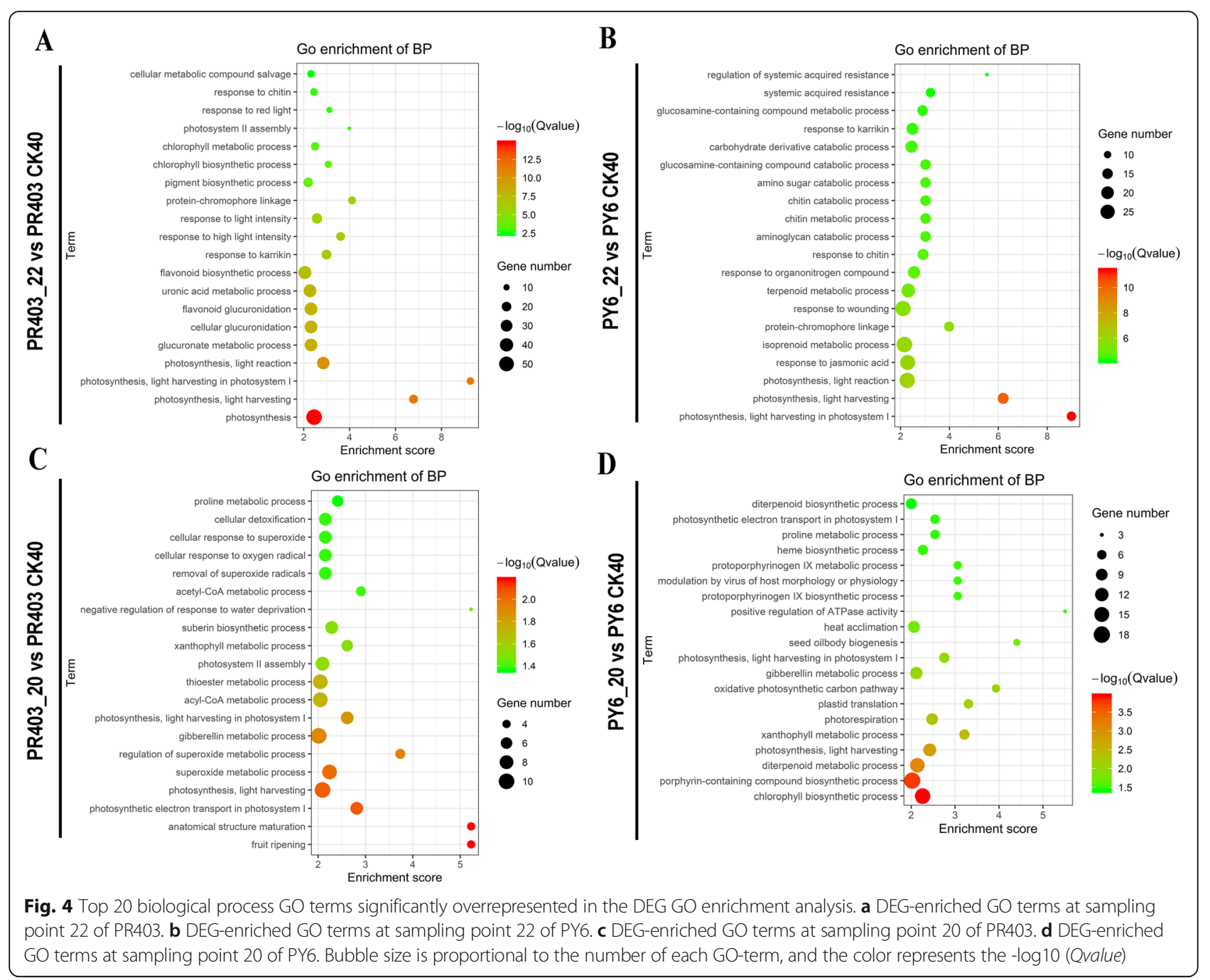

$1.0 \times 10^{-3}$ ), and royalblue (45 genes; $\mathrm{R}^{2}=0.68, P=4.0 \times$ $10^{-5}$ ) modules were considered strongly correlated with MDA (Fig. 5b, d). The genes in which the modules were clustered showed strong correlation with their respective modules (Fig. S7A, B). Furthermore, hub genes were identified based on the following cut-off thresholds: GS $>0.60$ and MM (Module Membership) $>0.80$ for the modules black, blue, green, and royalblue, and GS $>0.5$ and MM > 0.8 for the modules grey 60 , red, and brown according to the threshold strengthening. After removing the genes with $\left|\log _{2} \mathrm{FC}\right|<1$, the expression of all the obtained hub genes was significantly differentially altered in PY6 and PR403 under drought stress and were highlighted in subsequent functional analyses (Fig. S8A-F; Table S3-1 7). Hereafter, we designated these hub genes as DEHGs.

\section{Functional enrichment analysis of hub genes correlated with $\mathrm{H}_{2} \mathrm{O}_{2}$ accumulation}

GO enrichment analysis was performed to functionally cluster the hub genes in the modules that were strongly correlated with $\mathrm{H}_{2} \mathrm{O}_{2}$. A total of 115 and 204 DEHGs were identified in the modules black and blue, respectively (Table S3-1, -2). In line with the abovementioned results, these hub genes were highly enriched in the GO terms involving chloroplast (GO:0009507); the chloroplast envelope (GO: 0009941); thylakoid (GO:0009579); chloroplast stroma (GO:0009570); chlorophyll binding (GO:0016168); photosynthesis (GO: GO:0015979); photosystem I (GO:0009522); photosynthesis, light harvesting (GO:0009765); and photosynthesis, light reaction (GO:0019684). These terms are related to photosynthesis $(Q v a l u e \leq 0.05)$ (Fig. S9A-B; Table S5-1, - 2). Interestingly, in agreement with previous reports [59], the expression of the majority of the DEHGs in the modules black and blue was downregulated without marked $\log _{2} \mathrm{FC}$ differences $\left(\left|\log _{2} \mathrm{FC}_{\mathrm{PY} 6}-\log _{2} \mathrm{FC}_{\mathrm{PR} 403}\right| \geq 1\right.$, $P<0.05)$ observed between PY6 and PR403 during drought treatment (Fig. 6a, b; Fig. S8A, B), implying that PY6 and PR403 have a common drought-responsive regulation and that their photosynthetic activities were inhibited in response to drought stress. 

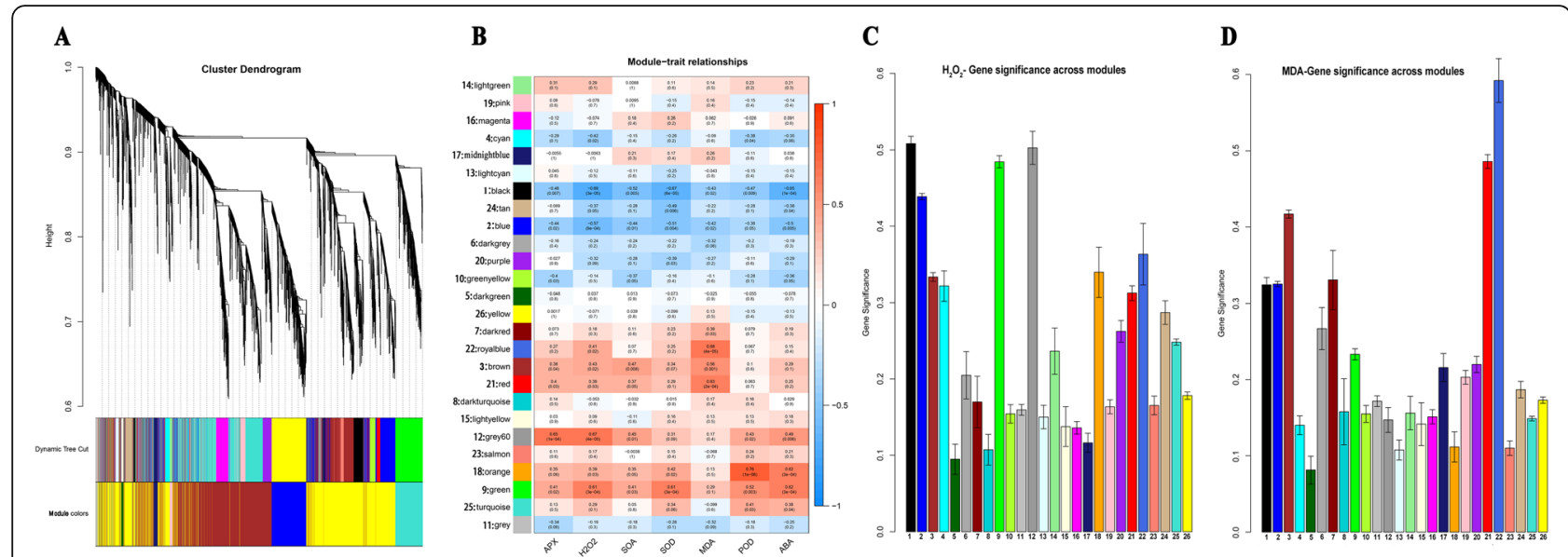

Fig. 5 WGCNA coexpression network and module-trait correlation analysis. a Hierarchical cluster tree showing coexpression modules identified via the Dynamic Tree Cut method. Different modules are marked with different colors. Each leaf in the tree represents one gene. The major tree branches constitute 26 modules and are labeled with different colors. b Correlations of physiological indicators with WGCNA modules. Each row corresponds to a module and is labeled with the same color as that in a. The columns correspond to physiological indicators. The color of each cell indicates the correlation coefficient between the module and physiological indicator (the top number in the cell represents the correlation coefficient, and bottom one in parentheses represents the $P$ value). c Correlations of $\mathrm{H}_{2} \mathrm{O}_{2}$ with WGCNA modules. $\mathbf{d}$ Correlations of $\mathrm{H}_{2} \mathrm{O}_{2}$ with WGCNA modules. The color bars with the numbers on the $\mathrm{X}$-axis in $(\mathbf{c})$ and $(\mathbf{d})$ designate the modules corresponding to those with numbers and names shown on left side of (b)

The module grey60 was enriched with 41 DEHGs (Table S3-3). The expression of most of these DEHGs was elevated under drought stress (Fig. S8C). Among these genes, eight showed differential expression with markedly different magnitudes at least at one sampling point between two genotypes, exhibiting a strong dynamic induction in response to drought treatment $\left(\left|\log _{2} \mathrm{FC}_{\mathrm{PY}}-\log _{2} \mathrm{FC}_{\mathrm{PR} 403}\right| \geq 1, P<0.05\right)$ (Fig. 6c; Table 2). The expression of Os01G0842500 and Os03G0273200, both of which encodes proteins that are similar to laccase and both of which were enriched in GO terms involving the apoplast (GO:0048046)/lignin catabolic process (GO:0046274)/cytoplasmic membrane-bounded vesicle (GO:0016023)/oxidoreductase activity (GO: 0016491), was consistently enhanced at two points of both PY6 and PR403 and showed higher magnitudes in PY6 (particularly at point 20) (Fig. 6c; Fig. S9C; Table 2; Table S5-3). Five genes (Os01G0702700, Os06G0131700, Os05G0543600, Os03G0433200, and Os04G0508500), which are annotated as transcription factors and mainly enriched in GO terms involving DNA binding (GO:0003677), sequence-specific DNA binding transcription factor activity (GO:0003700), and response to freezing (GO:0050826), were significantly

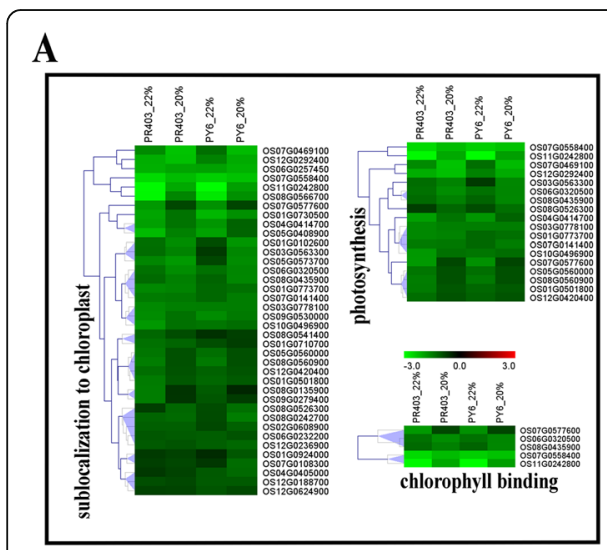

black module

B

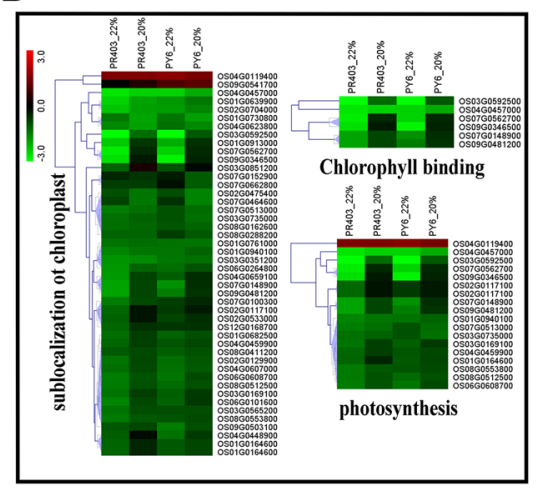

blue module

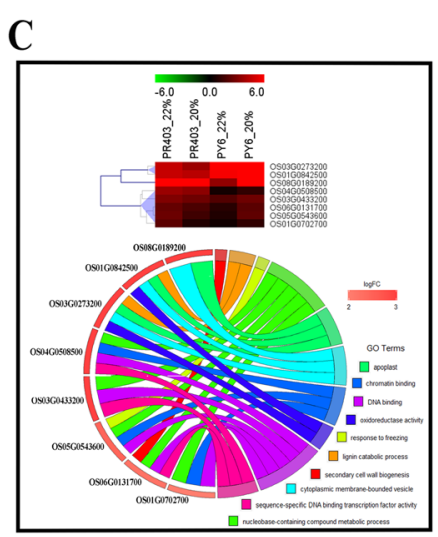

grey 60 module

Fig. 6 Heatmap and circular plot showing the altered expression and functional annotations of hub genes in the modules correlated with $\mathrm{H}_{2} \mathrm{O}_{2}$ accumulation. $\mathbf{a}-\mathbf{b}$ Expression patterns of hub genes of the modules black and blue showing that they are related to photosynthesis of rice plants. c Expression patterns and GO enrichment of hub genes of the module grey60. Different colors represent hub gene-enriched corresponding GO terms. All the data used in the analysis were subjected to log2 transformation 
Table 2 Differentially expressed hub genes identified in the module grey60

\begin{tabular}{|c|c|c|c|c|c|c|}
\hline Gene_id & PR403_22 ${ }^{\mathrm{a}}$ & PR403_20 & PY6_22 & PY6_20 & Description & GO enrichment \\
\hline Os01G0842500 & $4.69^{c}$ & 4.40 & 5.85 & 8.49 & Similar to Laccase (EC 1.10.3.2). & $\begin{array}{l}\text { GO:0005507, GO:0009809, GO:0016722, } \\
\text { GO:0046274, GO:0048046, GO:0052716, } \\
\text { GO:0055114 }\end{array}$ \\
\hline Os03G0273200 & 4.65 & 3.70 & 6.79 & 9.00 & Similar to Laccase (EC 1.10.3.2). & $\begin{array}{l}\text { GO:0005507, GO:0009809, GO:0016722, } \\
\text { GO:0046274, GO:0048046, GO:0052716, } \\
\text { GO:0055114 }\end{array}$ \\
\hline Os01G0702700 & 2.20 & 0.81 & 0.41 & 1.16 & $\begin{array}{l}\text { Similar to Transcription factor MYB86 } \\
\text { (Myb-related protein 86) (AtMYB86) } \\
\text { (Myb homolog 4) (AtMyb4). }\end{array}$ & GO:0003677 \\
\hline Os05G0543600 & 2.56 & 1.51 & 0.79 & 2.28 & Similar to Myb-related protein. & GO:0003677 \\
\hline Os04G0508500 & 3.65 & 3.34 & 0.50 & 1.18 & $\begin{array}{l}\text { MYB family transcription factor, putative, } \\
\text { expressed. }\end{array}$ & GO:0003677 \\
\hline Os06G0131700 & 2.38 & 1.80 & 0.98 & 2.16 & Similar to NAM protein. & GO:0003677, GO:0005634, GO:0006355 \\
\hline Os08G0189200 & 7.90 & 7.40 & 3.48 & 6.64 & Germin-like protein 8-3, Disease resistance & $\begin{array}{l}\text { GO:0005618, GO:0030145, GO:0033609, } \\
\text { GO:0045735, GO:0046564, GO:0048046 }\end{array}$ \\
\hline Os03G0433200 & 3.04 & 2.62 & 1.94 & 2.63 & $\begin{array}{l}\text { SHR transcription factor, Regulation of the } \\
\text { number of cortex cell layers in the root, } \\
\text { Coordination of stomatal patterning }\end{array}$ & $\begin{array}{l}\text { GO:0003700, GO:0005634, GO:0006355, } \\
\text { GO:0008356, GO:0009956, GO:0043565, } \\
\text { GO:0045930, GO:0048366, GO:0055072 }\end{array}$ \\
\hline
\end{tabular}

Notes: ${ }^{\text {a }}$ represents the samples collected at the $22 \%$ of soil moisture content; ${ }^{b}$ represents the samples collected at the $20 \%$ of soil moisture content; ${ }^{c}$ the value represents the $\log 2$ transformation of the fold change of the expression

consistently induced to a certain extent at point 22 and were downregulated at point 20 in PR403, whereas they were significantly induced only at point 20 in PY6, with the exception of Os03G0433200 (Fig. 6c; Table 2; Table S5-3). Os01G0702700, Os05G0543600, and Os04G0508500 are MYB family TFs, and Os06G0131700 is a NAC TF. A recent study suggested that Os06G0131700 (OsSWN1) is related to secondary cell wall formation [60], implying that secondary cell wall formation may play a certain role in drought stress resistance. In addition, Os08g0189200 is annotated as a gene encoding Germin-like protein 8-3 and was suggested to be involved in disease resistance [61]. A different upregulation pattern of this gene was observed in PY6 and PR403 in response to drought stress (Fig. 6c; Table 2).

A total of 96 hub genes were identified in the module green, including 36 downregulated and 60 upregulated genes in response to drought (Fig. S8D; Table S3-4). GO enrichment analysis revealed that the majority of the overrepresented $\mathrm{GO}$ terms were related to oxidation-reduction process (GO:0055114), response to salt stress (GO:0009651), carbohydrate metabolic process (GO:0005975), biosynthetic process (GO: 0009058), and metabolic process (GO:0008152). These hub genes were sublocalized to distinct cellular components and have various molecular functions (Fig. S9D; Table S5-4). However, with the exception of iron-sulfur cluster binding (GO:0051536), no GO term was significantly overrepresented in our analysis (Table S5-4). It is worth noting that only 1 hub gene, OS04G0685300, which encodes a protein with harpin-induced 1 domain, showed significant differential expression with a greater upregulation magnitude at two points of PY6 (2.03/2.67) compared with those in PR403 (Table S3-4).

Taken together, the functional annotation of the hub genes identified in the $\mathrm{H}_{2} \mathrm{O}_{2}$-related modules suggested that both PY6 and PR403 experienced inhibition of drought-triggered photosynthetic activity, leading to the accumulation of $\mathrm{H}_{2} \mathrm{O}_{2}$ in the rice plants. The hub genes with highly differential expression identified in the module grey60 may be responsible for the accumulation of $\mathrm{H}_{2} \mathrm{O}_{2}$ in rice plants.

\section{Functional enrichment analysis of hub genes correlated with MDA accumulation}

MDA is an important indicator of membrane lipid peroxidation. The modules red, brown, and royalblue were strongly correlated with MDA under drought treatment; there were 303, 304, and 13 hub genes identified in these modules, respectively (Table S3-5 - 7). For the module red, GO analysis revealed that the hub genes are involved in biotic stress and defense responses (Fig. S10A; Table S5-5). Among these hub genes, 29 showed differential expression at least at one sampling point between two genotypes $\left(\left|\log 2 \mathrm{FC}_{\mathrm{PY}}-\log 2 \mathrm{FC}_{\mathrm{PR} 403}\right| \geq 1, \quad P<0.05\right)$ (Fig. 7a; Table 3). Strikingly, most of them consistently showed similar expression patterns in both genotypes, with elevated transcripts at point 22 , after which the expression levels then declined to levels that were comparable to or even lower than those of their respective untreated controls (Fig. S8E; Table 3). GO analysis revealed that these genes were involved in biotic stress responses; for example, the GO terms involving defense response (GO:0006952), response to biotic stimulus 


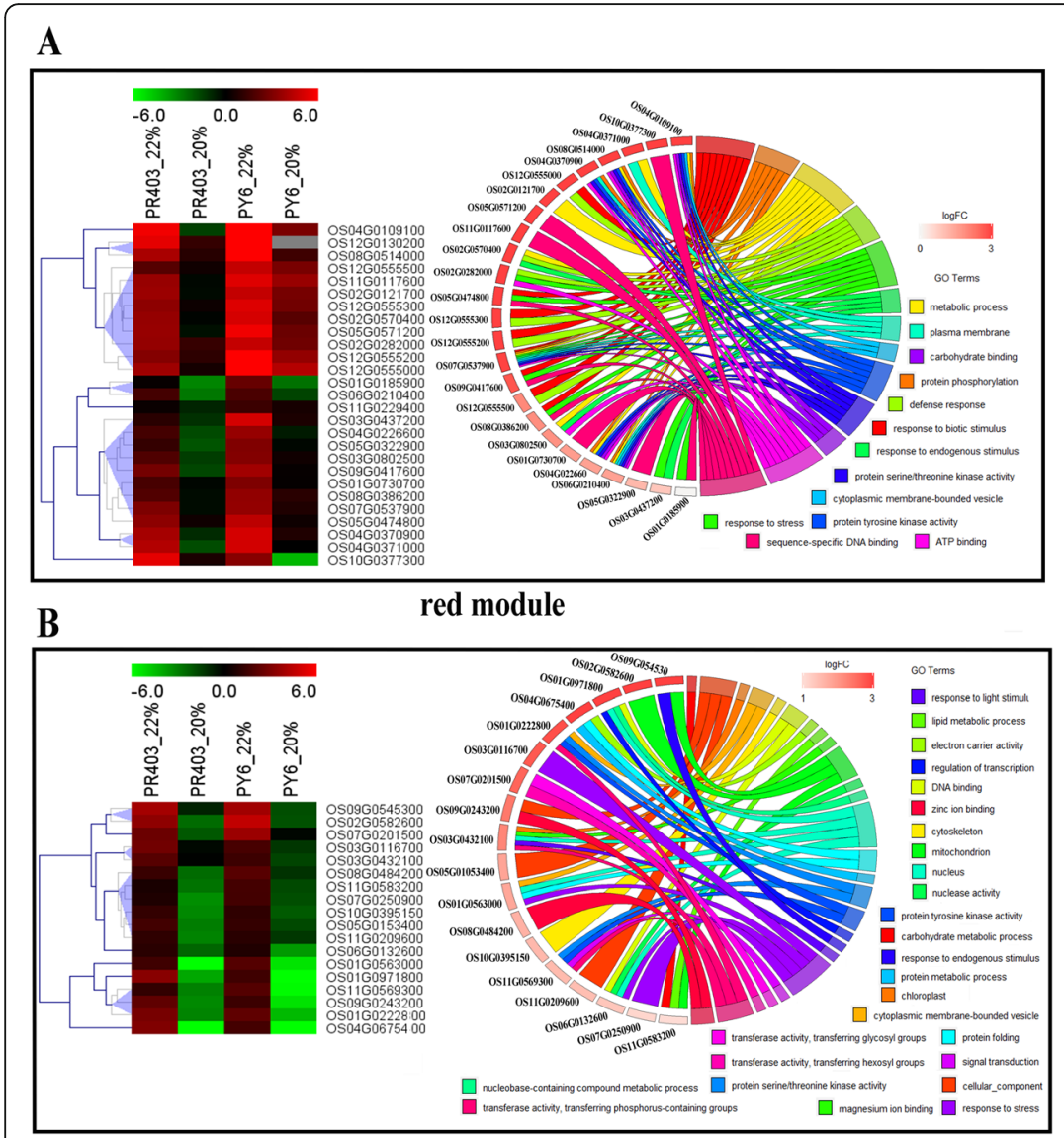

brown module
C

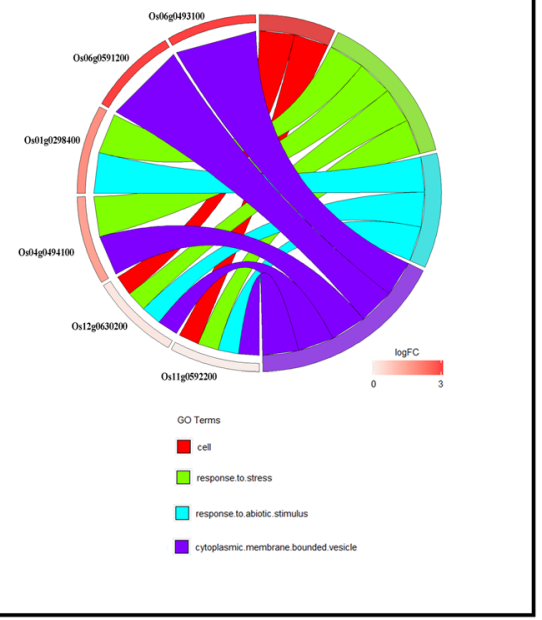

royalblue module

Fig. 7 Heat map and circular plot showing the altered expression and functional annotations of hub genes in the modules correlated with MDA accumulation. a Expression patterns and $\mathrm{GO}$ enrichment of hub genes of the module red. $\mathbf{b}$ Expression patterns and $\mathrm{GO}$ enrichment of hub genes of the module brown. c Expression patterns and GO enrichment of hub genes of the module royalblue. All the data used in the analysis were subjected to log2 transformation. Different colors represent hub gene-enriched corresponding GO terms

(GO:0009607), response to stress (GO:0006950), and response to endogenous stimulus (GO:0009719) were overrepresented in the results (Fig. 7a; Table S5-5). Of these genes, 8 were WRKY family TFs (Fig. 7a; Table 3). Specifically, OsWRKY70 (Os05g0474800) and OsWRKY76 (Os09g0417600) have been suggested to participate in ABA signaling and the biotic stress response [34, 62]. OS01g0185900 and OS05g0322900 exhibited relatively higher upregulated expression at point 22, whereas two other genes, OS05g0571200 and OS11G0117600, had relatively higher expression at point 20 of PY6 than at PR403 (Fig. 7a; Table 3). Considering the accumulation of MDA in PY6 and PR403, these results implied that four genes may be positively correlated with the overaccumulation of MDA and lead to the death of PY6 under drought stress. Moreover, PR-10a (OS12g0555300), which functions at the downstream of jasmonic acid pathway and is positively regulated by WRKY TFs in response to drought and high-salt stresses $[38,63]$, and three other PR-10 protein family hub genes
(Os12g0555200, Os12g0555500, and Os12g0555000), which were annotated as genes encoding probenazoleinducible PBZ1 proteins and are involved in disease resistance, exhibited a higher magnitude of upregulated expression at two sampling points of PY6 compared with PR403 (Fig. 7a; Table 3). BSR-d1/ZFP36 (Os03g0437200), which encodes a $\mathrm{C}_{2} \mathrm{H}_{2}$-type zinc finger protein, participates in ABA-OsMPK transduction, which results in the accumulation of $\mathrm{H}_{2} \mathrm{O}_{2}[64,65]$. Similarly, this gene also had a higher upregulated expression in PY6 than in PR403 (Table 3). In addition, the expression of OS02g0121700 and OS02g0570400 was dramatically induced in PY6 during the whole period of drought treatment, whereas these genes were significantly induced only at point 22 of PR403, after which their expression levels decreased to those of the untreated control (Fig. 7a; Table 3). These genes encode a terpenoid synthase domain-containing protein and ent-kaurene synthase 1A, which are involved in gibberellin (GA) biosynthesis, suggesting a role of GA in the 
Table 3 Differentially expressed hub genes identified in the module red

\begin{tabular}{|c|c|c|c|c|c|c|}
\hline Gene_id & ${ }^{\text {PR403_22 }}{ }^{\mathrm{a}}$ & PR403_20 & PY6_22 & PY6_20 & Description & GO enrichment \\
\hline OS01G0730700 & $1.71^{\mathrm{c}}$ & -0.16 & 3.00 & 0.15 & WRKY transcription factor 14 (WRKY14). & GO:0003700, GO:0006355, GO:0043565 \\
\hline OS05G0474800 & 3.36 & 0.38 & 4.46 & 0.36 & WRKY transcription factor 70. & GO:0003700, GO:0006355, GO:0043565 \\
\hline OS09G0417600 & 2.76 & -1.65 & 3.82 & 0.17 & WRKY transcription factor 76. & $\begin{array}{l}\text { GO:0003700, GO:0006355, GO:0009617, } \\
\text { GO:0009620, GO:0009751, GO:0010200, } \\
\text { GO:0043565 }\end{array}$ \\
\hline OS08G0386200 & 1.95 & -0.28 & 3.46 & 0.99 & WRKY transcription factor 69. & GO:0003700, GO:0006355, GO:0043565 \\
\hline OS01G0185900 & 0.19 & -3.15 & 2.43 & -2.67 & Similar to WRKY 1 (Fragment). & GO:0003700, GO:0006355, GO:0043565 \\
\hline OS05G0322900 & 1.25 & -1.98 & 3.04 & -0.17 & Similar to WRKY transcription factor 45. & $\begin{array}{l}\text { GO:0003700, GO:0009862, GO:0009864, } \\
\text { GO:0010200, GO:0043565, GO:0045892, } \\
\text { GO:0050832, GO:1900056 }\end{array}$ \\
\hline OS05G0571200 & 3.60 & -0.37 & 5.70 & 2.53 & Similar to WRKY transcription factor 19. & GO:0003700, GO:0006355, GO:0043565 \\
\hline OS11G0117600 & 3.59 & -0.18 & 5.29 & 3.60 & $\begin{array}{l}\text { Similar to WRKY transcription factor } 50 \\
\text { (Fragment). }\end{array}$ & GO:0003700, GO:0006355, GO:0043565 \\
\hline OS03G0437200 & 1.00 & -1.14 & 5.03 & 0.67 & $\begin{array}{l}\mathrm{C} 2 \mathrm{H} 2 \text {-type zinc finger protein, Abscisic } \\
\text { acid-induced antioxidant defence, Water } \\
\text { stress and oxidative stress tolerance }\end{array}$ & $\begin{array}{l}\text { GO:0003700, GO:0005634, GO:0006355, } \\
\text { GO:0043565, GO:0044212, GO:0046872 }\end{array}$ \\
\hline OS10G0377300 & 5.42 & 0.66 & 3.21 & -4.34 & $\begin{array}{l}\text { Similar to Homeobox-leucine zipper } \\
\text { protein HOX8. }\end{array}$ & $\begin{array}{l}\text { GO:0003700, GO:0005634, GO:0006355, } \\
\text { GO:0043565 }\end{array}$ \\
\hline OS12G0555200 & 3.14 & 1.10 & 6.30 & 3.50 & $\begin{array}{l}\text { Similar to Probenazole-inducible } \\
\text { protein PBZ1. }\end{array}$ & $\begin{array}{l}\text { GO:0004864, GO:0004872, GO:0005634, } \\
\text { GO:0005737, GO:0006952, GO:0009607, } \\
\text { GO:0009738, GO:0010427, GO:0043086, } \\
\text { GO:0080163 }\end{array}$ \\
\hline OS12G0555500 & 2.03 & 0.42 & 4.62 & 2.85 & $\begin{array}{l}\text { Pathogen resistance protein PBZ1,C } 17 \\
\text { kDa RNase, Disease resistance }\end{array}$ & $\begin{array}{l}\text { GO:0004864, GO:0004872, GO:0005634, } \\
\text { GO:0005737, GO:0006952, GO:0009607, } \\
\text { GO:0009738, GO:0010427, GO:0043086, } \\
\text { GO:0080163 }\end{array}$ \\
\hline OS12G0555000 & 3.75 & 0.52 & 6.81 & 4.27 & $\begin{array}{l}\text { Similar to Probenazole-inducible } \\
\text { protein PBZ1. }\end{array}$ & $\begin{array}{l}\text { GO:0004864, GO:0004872, GO:0005634, } \\
\text { GO:0005737, GO:0009738, GO:0010427, } \\
\text { GO:0043086, GO:0080163 }\end{array}$ \\
\hline OS12G0555300 & 3.35 & 0.35 & 5.23 & 2.70 & $\begin{array}{l}\text { Similar to Pathogenesis-related } \\
\text { protein PR-10a. }\end{array}$ & GO:0006952, GO:0009607 \\
\hline OS02G0282000 & 3.52 & 1.30 & 4.64 & 2.65 & NB-ARC domain containing protein. & GO:0043531 \\
\hline OS01G0134700 & 2.02 & -0.96 & 3.78 & 0.29 & $\begin{array}{l}\text { Calmodulin binding protein-like family } \\
\text { protein. }\end{array}$ & $\begin{array}{l}\text { GO:0002229, GO:0003700, GO:0005516, } \\
\text { GO:0005634, GO:0006355, GO:001011, } \\
\text { GO:0010224, GO:0043565, GO:0071219, } \\
\text { GO:0080142, GO:1900426 }\end{array}$ \\
\hline OS01G0508100 & 2.48 & -1.35 & 4.13 & 0.89 & $\begin{array}{l}\text { Ferritin/ribonucleotide reductase-like } \\
\text { family protein. }\end{array}$ & GO:0010112 \\
\hline OS02G0121700 & 3.68 & -0.23 & 4.63 & 2.82 & $\begin{array}{l}\text { Terpenoid synthase domain } \\
\text { containing protein. }\end{array}$ & $\begin{array}{l}\text { GO:0000287, GO:0009507, GO:0010333, } \\
\text { GO:0016114, GO:0034007, GO:0042742, } \\
\text { GO:0043693 }\end{array}$ \\
\hline OS02G0570400 & $3.55^{\mathrm{C}}$ & -0.11 & 5.06 & 2.08 & Similar to Ent-kaurene synthase $1 \mathrm{~A}$. & $\begin{array}{l}\text { GO:0000287, GO:0006952, GO:0008152, } \\
\text { GO:0010333, GO:0034277 }\end{array}$ \\
\hline OS03G0103300 & 3.42 & -1.76 & 4.33 & 1.68 & $\begin{array}{l}\text { Hybrid proline- or glycine-rich protein, } \\
\text { Control of low-temperature germinability, } \\
\text { Pre-harvest sprouting resistance }\end{array}$ & $\begin{array}{l}\text { GO:0005783, GO:0009506, GO:0009707, } \\
\text { GO:0050832 }\end{array}$ \\
\hline OS04G0226600 & 1.63 & -1.76 & 3.56 & -0.63 & Similar to OSIGBa0135L04.3 protein. & $\begin{array}{l}\text { GO:0004674, GO:0005524, GO:0005886, } \\
\text { GO:0006468, GO:0006952, GO:0009506 }\end{array}$ \\
\hline OS05G0368000 & 4.03 & -1.95 & 5.31 & 0.50 & Non-protein coding transcript. & GO:0010112 \\
\hline OS07G0537900 & 2.97 & -0.27 & 3.49 & 0.77 & Similar to SRK3 gene. & $\begin{array}{l}\text { GO:0004674, GO:0005524, GO:0005886, } \\
\text { GO:0006468, GO:0006952, GO:0009506, } \\
\text { GO:0016021 }\end{array}$ \\
\hline OS06G0210400 & 1.63 & -2.81 & 1.79 & -1.61 & $\begin{array}{l}\text { Concanavalin A-like lectin/glucanase, } \\
\text { subgroup domain containing protein. }\end{array}$ & $\begin{array}{l}\text { GO:0004674, GO:0005524, GO:0006468, } \\
\text { GO:0016021, GO:0030246 }\end{array}$ \\
\hline OS04G0109100 & 5.74 & -1.52 & 8.78 & 2.92 & Concanavalin A-like lectin/glucanase & GO:0004674, GO:0005524, GO:0006468, \\
\hline
\end{tabular}


Table 3 Differentially expressed hub genes identified in the module red (Continued)

\begin{tabular}{lllllll}
\hline Gene_id & PR403_22 & PR403_20 & PY6_22 & PY6_20 & Description & GO enrichment \\
\hline OS04G0370900 & 3.91 & -1.01 & 5.03 & 0.77 & Similar to H0607F01.5 protein. & GO:0030246 \\
OS04G0371000 & 4.22 & -1.80 & 4.98 & 0.05 & Four-helical cytokine family protein. & $\begin{array}{l}\text { GO:0004672, GO:0005509, GO:0005515, } \\
\text { GO:0005524, GO:0006468, GO:0016021, } \\
\text { GO:0030247 }\end{array}$ \\
OS08G0514000 & 4.02 & 0.95 & $\mathbf{6 . 6 1}$ & 1.49 & $\begin{array}{l}\text { Protein kinase, catalytic domain } \\
\text { containing protein. }\end{array}$ & $\begin{array}{l}\text { GO:0030247 } \\
\text { GO:0004672, GO:0005524, GO:0006468, }\end{array}$ \\
OS03G0802500 & 1.90 & -1.45 & 3.13 & 0.42 & $\begin{array}{l}\text { ATPase, AAA-type, C core domain } \\
\text { containing protein. }\end{array}$ & GO:0005524, GO:0016021 \\
\hline
\end{tabular}

Notes: ${ }^{a}$ represents the samples collected at the $22 \%$ of soil moisture content; ${ }^{b}$ represents the samples collected at the $20 \%$ of soil moisture content; ${ }^{c}$ the value represents the $\log 2$ transformation of the fold change of the expression

drought stress response in rice. OS04g0109100, a gene annotated as concanavalin A-like lectin/glucanase, had a similar expression pattern in both genotypes.

In total, 18 and 13 DEHGs were identified in the modules brown and royalblue, respectively (Fig. 7b, c; Table 4; Table S4). GO analysis revealed that the DEHGs in the module brown play different roles in various biological processes, including response to stress (GO:0006950), response to endogenous stimulus (GO:0009719), protein phosphorylation (GO:0006468), protein metabolic process (GO:0019538), lipid metabolic process (GO: 0006629), carbohydrate metabolic process (GO: 0005975), protein folding (GO:0005515), regulation of transcription (GO:0006355), and signal transduction (GO:0007165) (Fig. 7b; Table S5-6). Nearly all of these genes were induced at point 22 and were repressed at point 20 in response to drought in both genotypes.
Although they have a similar expression pattern, a different extent of downregulation was observed in several hub genes; for example, OS11G0569300, OS01G0971800, OS04G0675400, and OS09G0243200 showed a lower extent of downregulation at point 20 of PY6, while OS07G0201500 and OS11G0209600 showed a lower extent of downregulation at point 20 of PR403 (Fig. 7b; Table S4). In the module royalblue, only one GO term was significantly overrepresented due to the relatively low number of DEHGs. The striking feature of the expression pattern of the hub genes was that their transcripts were dramatically induced by drought at point 22 of PY6, and their levels were also slightly reduced at the next sampling point. Interestingly, Os04g0494100 and Os11g0592200 were annotated as genes encoding PR3 and PR4 family proteins, which have plant chitinase activity. Os03g0663600 and

Table 4 Differentially expressed hub genes identified in the module royalblue

\begin{tabular}{|c|c|c|c|c|c|c|}
\hline Gene_id & PR403_22 $^{\mathrm{a}}$ & PR403_20 ${ }^{b}$ & PY6_22 & PY6_20 & Description & GO enrichment \\
\hline Os01g0298400 & $1.91^{\mathrm{c}}$ & -0.31 & 3.15 & 0.57 & $\begin{array}{l}\text { Myb transcription factor domain } \\
\text { containing protein. }\end{array}$ & GO:0003677 \\
\hline Os01g0392600 & -0.81 & -2.14 & 2.14 & -2.02 & Conserved hypothetical protein. & \\
\hline Os03g0663600 & 1.87 & 0.68 & 4.31 & 2.03 & $\begin{array}{l}\text { Similar to Pathogenesis-related } \\
\text { thaumatin-like protein. }\end{array}$ & \\
\hline Os12g0630200 & 0.44 & -2.47 & 2.76 & -0.64 & $\begin{array}{l}\text { Thaumatin } \% 2 C \text { pathogenesis- } \\
\text { related family protein. }\end{array}$ & \\
\hline Os11g0592200 & 0.33 & -2.22 & 2.14 & -1.30 & $\begin{array}{l}\text { Similar to Chitin-binding allergen } \\
\text { Bra r } 2 \text { (Fragments). }\end{array}$ & GO:0042742, GO:0050832 \\
\hline Os04g0494100 & 1.63 & 0.65 & 4.83 & 1.19 & Similar to Chitinase. & $\begin{array}{l}\text { GO:0000272, GO:0004568, GO:0005618, } \\
\text { GO:0006032, GO:0006952, GO:0008061, } \\
\text { GO:0009611, GO:0009617, GO:0010262, } \\
\text { GO:0016998 }\end{array}$ \\
\hline Os06g0487620 & 1.97 & 1.70 & 2.31 & 0.98 & Conserved hypothetical protein. & \\
\hline Os06g0493100 & 5.99 & 0.26 & 5.93 & 1.68 & Conserved hypothetical protein. & GO:0016021 \\
\hline Os06g0586300 & -0.01 & 1.13 & 5.08 & 3.48 & Hypothetical protein. & \\
\hline Os06g0588450 & -1.00 & -1.82 & 4.64 & 2.74 & Hypothetical conserved gene. & \\
\hline Os06g0591200 & 4.28 & -2.17 & 8.51 & 4.08 & Conserved hypothetical protein. & \\
\hline
\end{tabular}

Notes: ${ }^{a}$ represents the samples collected at the $22 \%$ of soil moisture content; ${ }^{b}$ represents the samples collected at the $20 \%$ of soil moisture content; ${ }^{c}$ the value represents the $\log 2$ transformation of the fold change of the expression 
Os12g0630200 are two genes encoding pathogenesisrelated thaumatin-like proteins, which are members of the PR5 family, suggesting that these genes played roles in the cross-talk between drought and biotic stresses in this study (Fig. 7c; Table 4; Table S5-7).

\section{Discussion}

In this study, CSSL PY6 was used to characterize a QTL locus, $d s s-1$, for its drought-sensitive phenotype and to investigate the impact of $d s s-1$ on the reprogramming of transcriptional profiles of PY6 in response to drought stress via RNA-seq and WGCNA.

\section{Drought-induced overaccumulation of $\mathrm{H}_{2} \mathrm{O}_{2}$ may lead to the sensitive phenotype of $d s s-1$}

Reactive oxygen species (ROS) (e.g., $\mathrm{O}^{2--}, \mathrm{H}_{2} \mathrm{O}_{2}, \mathrm{OH}^{*}$, ${ }^{1} \mathrm{O}_{2}$ ) are unavoidable toxic byproducts of plants generated in response to abiotic/biotic stresses [66]. The overaccumulation of ROS in cells causes severe oxidative damage to membranes (lipid peroxidation), proteins, RNA, and DNA molecules [67]. Higher plant has thus evolved dedicated scavenging pathways to protect themselves from ROS toxicity, including pathways involving detoxifying enzymes such as CAT, SOD, POD, and APX, as well as the antioxidant ascorbate-glutathione (GSH) cycle $[19,68]$. In this study, significant overaccumulation of $\mathrm{H}_{2} \mathrm{O}_{2}$ was detected in PY6 compared with PR403 during drought treatment (Fig. 2a, b). Regarding the drought-sensitive phenotype of PY6, which was afforded by the introgression of $d s s-1$ into the background of PR403, our results suggested that important relationships were evident among dss- $1, \mathrm{H}_{2} \mathrm{O}_{2}$ accumulation, and the sensitive phenotype. That is, we speculated that the introgression of $d s s-1$ might directly or indirectly lead to the overaccumulation of $\mathrm{H}_{2} \mathrm{O}_{2}$ in rice cells. The overaccumulation of $\mathrm{H}_{2} \mathrm{O}_{2}$ causes oxidative damage to the cell membrane (lipid peroxidation), which was reflected by the measurements of MDA and others, subsequently leading to the death of rice plants under severe drought stress conditions.

\section{Photosynthesis regulation, a common regulatory process for rice in response to drought stress}

Chloroplasts are important organelles for the fixation of light energy necessary for the biological activity of higher plant and all other life forms in our biosphere [69]. Beyond their normal role in photosynthesis, increasing evidence suggests that chloroplasts are targeted organelles that are involved in environmental stress responses by positively or passively tuning photosynthetic activity to adapt to environmental changes [24]. According to the GO enrichment analysis, the drought-induced DEGs identified either in PR403 or PY6 were mainly enriched in photosynthesis-related GO terms (Fig. 4; Figs. S4; S5).
Additionally, the analysis of differentially expressed hub genes that clustered in the modules black and blue showed that a similar set of GO terms that are related to the photosynthesis process in chloroplasts were overrepresented (Fig. S9A, B). In terms of the altered expression during drought treatment, in line with the results of previous studies [59], the majority of hub genes clustering in the modules black and blue consistently declined to a similar extent in both PY6 and PR403 (Fig. S8A, B), implying that the photosynthetic activity of both genotypes was dramatically inhibited in response to drought stress. The inhibition of photosynthesis reduces the utilization of absorbed light energy; excess light energy results in the generation of toxic ROS [17]. Therefore, photosynthetic activity regulation could be a common regulatory process that is shared among diverse varieties for the adaptation of rice in response to environmental stresses. The inhibition of photosynthesis in both genotypes of this study might be correlated to the accumulation of $\mathrm{H}_{2} \mathrm{O}_{2}$ in rice cells. The differential accumulation of $\mathrm{H}_{2} \mathrm{O}_{2}$ in the leaves of PY6 and PR403 results from the effects of the introgression of $d s s-1$ into PR403 on the different expression changes of the hub genes identified in the related modules. Furthermore, previous studies considered that the improvement of photosynthetic capability was important for drought resistance of plants [42]. However, in this study, no significant expression difference was observed in the photosynthesis-related DEGs identified in either PR403 or PY6, suggesting that the $d s s$-1-induced drought-sensitive phenotype was not caused by the severe inhibition of photosynthesis but, rather, by overaccumulation of $\mathrm{H}_{2} \mathrm{O}_{2}$.

\section{ROS-correlated hub genes that were differentially regulated in response to drought stress}

Transcription factors are key players in the regulatory networks of plants in response to unfavorable stresses. The MYB family is one of the largest transcription factor families in plants; its members have a conserved MYBbinding domain, and the MYB family TFs have been shown to be induced by $\mathrm{H}_{2} \mathrm{O}_{2}$ in soybean and play essential roles in response to abiotic stress [70, 71]. In this study, five DEGs annotated as transcription factors, including 3 MYBs, $1 \mathrm{NAD}$, and 1 SHR, were highly clustered in the module grey60, which was statistically correlated with the accumulation of $\mathrm{H}_{2} \mathrm{O}_{2}$ during drought stress. Among the genes, Os01G0702700, Os05G0543600, and Os04G0508500 are MYB family transcription factors and showed a higher magnitude of upregulation at point 22 of PR403. Considering the relatively low accumulation of $\mathrm{H}_{2} \mathrm{O}_{2}$ and MDA in PR403, MYB family TFs may be induced by drought stress or other signaling messengers and may trigger the activation of downstream components of drought-responsive 
signaling pathways. This action may also include the upregulation of stress-responsive genes involved in increasing the content of osmo-protectants and the activities of POD, CAT, and SOD, as well as maintaining a sublethal content of $\mathrm{H}_{2} \mathrm{O}_{2}$ in plant cells during drought stress, suggesting that the MYB TFs identified here were positively correlated with drought resistance (Figs. 2 and 6c; Table 2) [72]. Moreover, WRKY TFs, a family of transcription factors involved in plant defense responses [73], were found to be strongly correlated with MDA and 8 differentially expressed WRKY TFs identified in the module red showed a higher degree of induction at point 22 and a lower level at point 20 in PY6 compared with PR403 (Table 3).

PR proteins are another group of proteins that is involved in plant defense responses [38]. In this study, four PR10 family encoding genes identified in the module red and three other families of PR proteins (PR3, PR4, and PR5) identified in the module royalblue exhibited similar altered expression patterns in response to drought stress. Among the identified PR-encoding genes, OS12G0555000 (RSOsPR10), a gene encoding the rice root-specific pathogenesis-related protein PBZ1, was previously reported to be induced by drought and salinity stresses, as well as jasmonic acid; however, it was strongly inhibited by salicylic acid [38, 63]. Besides, the expression of RSOSPR10 was postulated to be repressed by SA-induced OSTGAs or OsWRKYs [38]. Our results suggested that cross-talk occurs between abiotic and biotic stress-responsive pathways during drought stress. Recent reports have revealed that some common components are shared by the signaling pathways involved in abiotic and biotic stress responses: the phytohormones abscisic acid, salicylic acid, and jasmonic acid; cis-acting regulatory elements; and components of protein kinase cascades [2]. Plants can rapidly respond to environmental changes by the synergistic or antagonistic regulation of cross-talk among pathways via their convergent nodes [2]. OsWRKY76 (OS09G0417600), a hub gene of the module red, encodes a group IIa WRKY TF in rice. It has been shown that overexpression of OsWRKY76 in rice plants suppresses a specific set of PR genes and increases the expression of abiotic stress-associated genes, which resulted in increased susceptibility to Magnaporthe oryzae (M. oryzae) and improved tolerance to chilling stress [34]. In addition, OsWRKY13 was suggested to regulate the antagonistic cross-talk between drought and disease resistance pathways via the repression of SNAC1 and WRKY45-1 [33]. Thus, the WRKY TF- and PR family protein-encoded genes identified here may act as convergent nodes and may be responsible for the cross-talk between abiotic and biotic stresses.

In addition, strikingly, Os01G0842500 and Os03G0273200, both of which were annotated as putative laccase-encoding genes, consistently exhibited elevated expression in both genotypes in response to drought. However, PY6 had a relatively higher upregulated expression at point 20 , implying that the overaccumulation of the transcripts of the genes for laccase may be correlated with the drought-sensitive phenotype of PY6. It has been demonstrated that a rice laccaseencoding gene, OsChi1, has been reported to play an important role in the ROS signaling pathway [74], suggesting that, in the present study, MYB TF-regulated laccases with differential expression are involved in the improvement of drought stress tolerance (Fig. 8). However, paradoxically, the overexpression of another laccase-encoding gene, OsChi1 (Os01g0827300), in Arabidopsis resulted in increased tolerance to drought and salinity stresses [74]. Thus, further study needs to be performed to explore the function of the laccases identified in the study and determine the discrepancy. Moreover, ZFP36 (OS03G0437200), a gene encoding a $\mathrm{C}_{2} \mathrm{H}_{2}$ zinc finger protein (Brs-d1), is a key factor for regulating rice cell redox status and is involved in $\mathrm{ABA}$-induced upregulation of the expression and activity of SOD and APX. Overexpression of ZFP36 enhanced tolerance to drought and oxidative stresses but reduced resistance to rice blast $[64,65]$. However, dramatically increased expression of ZFP36 was exhibited in the drought-sensitive genotype PY6, suggesting that this gene may play a synergistic role in the regulatory network with WRKY TFs and PRs in rice plants in response to drought stress.

\section{Impact of dss-1 on the reprogramming of hub genes in response to drought stress}

Taken together, a working model was proposed here to depict the possible $d s s-1$-mediated mechanism of rice plants in response to drought stress at the transcriptional level (Fig. 8). When rice is exposed to severe drought stress, stress-induced dehydration of rice plants and stomatal closure of leaf guard cells result in the inhibition of photosynthesis activity and the enhancement of ABA biosynthesis. The inhibition of photosynthesis reduces the utilization of absorbed light energy and results in the generation of toxic ROS, including $\mathrm{H}_{2} \mathrm{O}_{2}$. Overaccumulation of ROS can cause oxidative stress damage to plant cells, leading to the drought-sensitive phenotype of rice. Drought stress signals, ABA accumulation, and $\mathrm{H}_{2} \mathrm{O}_{2}$ signals are integrated and perceived by rice cells and trigger the activation of responsive signaling pathways. First, the expression of a series of TFs, including MYBs, WRKYs, ZFP36, NACs, etc., was differentially reprogrammed, and these TFs induced cross-talk between drought and biotic stress responses. Induction of the TFs then regulates the expression of downstream responsive genes, including a number of PR family proteins and laccases, to mobilize multiple aspects 


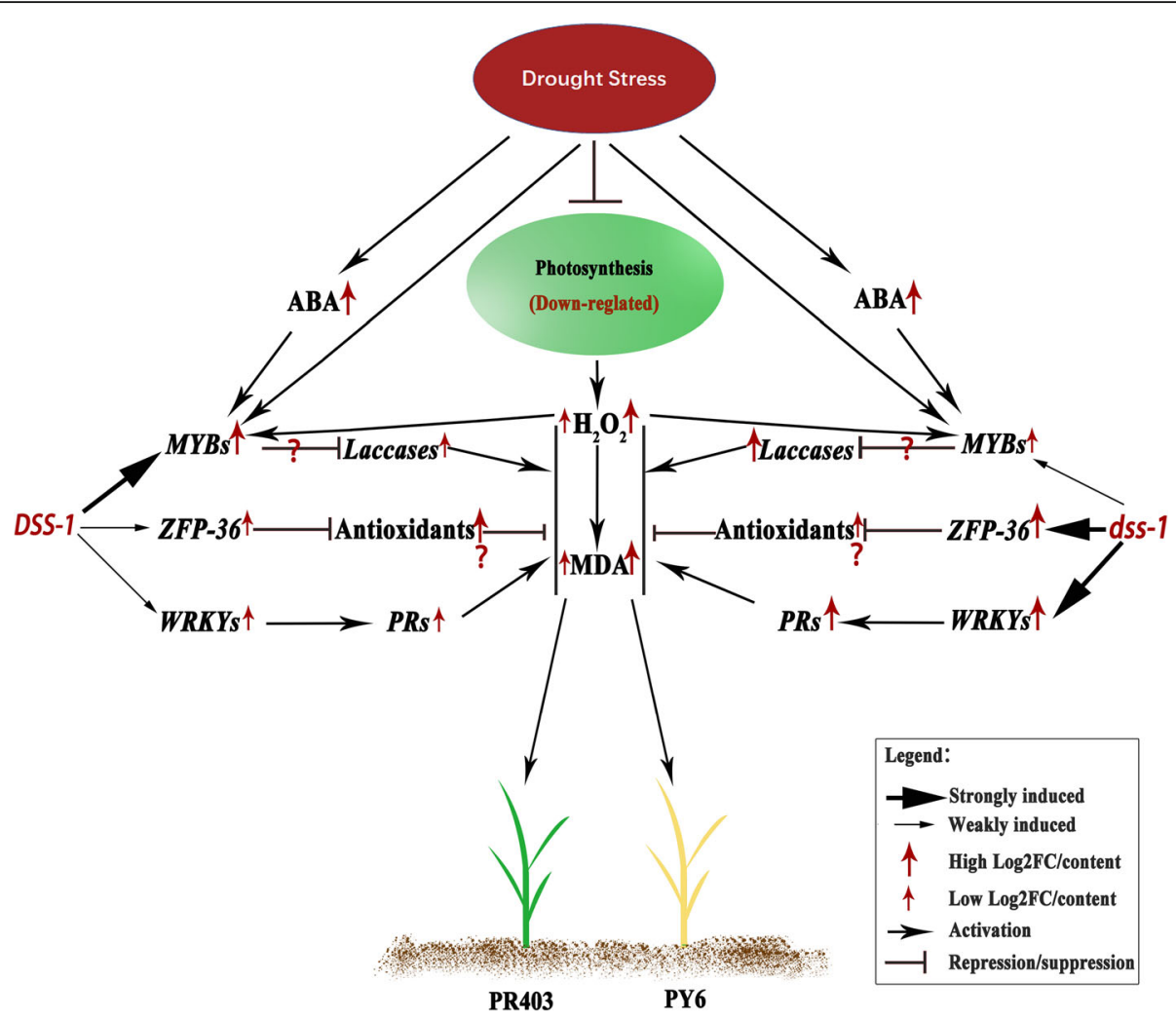

Fig. 8 Possible role of $d s s-1$ in reprogramming the transcriptional profiles of rice in response to drought stress. When rice is exposed to severe drought stress, the inhibition of photosynthesis leads to the overaccumulation of toxic ROS, including $\mathrm{H}_{2} \mathrm{O}_{2}$. $A B A$ and $\mathrm{H}_{2} \mathrm{O}_{2}$ signals are integrated and perceived and trigger the activation of responsive signaling pathways. First, the transcription factors, MYBs, WRKYs, ZFP36, NACs, etc., was differentially reprogrammed. The downstream responsive genes, including a number of PR family proteins and laccases, are then regulated to respond to drought stress, and ameliorating the drought-induced damage of rice. The introgressed dss-1 may exert its effect on the hub genes and differentially alter their expression, therefore leading to the drought-resistant and -sensitive variation of rice

of in vivo resources to respond to drought stress, and eventually reducing the content of ROS in plant cells and ameliorating the drought-induced sensitive phenotype of rice. The introgressed $d s s-1$ may exert its effect on the hub genes that were identified in our study to differentially alter their expression, therefore leading to the differential accumulation of ROS between drought-resistant and drought-sensitive varieties. The differential activation of ROS-related responsive pathways could cause the differences between the drought resistance/sensitive phenotypes of rice varieties.

In this study, we used a drought sensitive plant material to fine map QTL $d s s-1$ and investigate its effect on the transcriptomic profiling of rice. In subsequent, we will identify a drought sensitive QTL allele from Lambayeque 1 by positional cloning; the drought tolerant allele of dss-1 from PR403 can also be isolated as well. They will then be studied for the elucidation of drought tolerant mechanisms and drought tolerant allele DSS-1 will be applied for rice breeding.

\section{Conclusion}

Taken together, our results suggest that chloroplasts are the targeted organelles for PR403 and PY6 in response to drought stress regulation. Functional annotation analysis of the hub genes in the modules that were strongly correlated with $\mathrm{H}_{2} \mathrm{O}_{2}$ and MDA accumulation during drought treatment provided us with a global picture of the impact of $d s s-1$ on the reprogramming of the regulatory mechanism(s) at the transcriptional level. Specifically, the differential expression patterns of $M Y B s$, WRKYS, PR protein encoding genes, ZFP36, etc., in both genotypes implied their roles in the variation in ROS and MDA accumulation, thereby resulting in the sensitive phenotype of rice in response to drought stress.

\section{Methods}

Plant materials, growth, and treatment conditions

PY6 was screened in a set of CSSLs that were constructed by Jijing Luo, Jianbin Liu, and Baiyang Yu by introgression of the genomic fragments of donor parent Lambayeque 1 into recurrent parent PR403. The 
parental lines, PR403 (Accession number: AAV003680) and Lambayeque 1 (Accession number: AAV003699) are deposited in Genetic resource bank of Shanghai Agricultural Biological Gene Center (http://seed.sagc.org.cn/ front/custom/enterViewResourceList.action?gerTypeId= 4028940b44ba2d910144ba3062d00002\&gerTypeCode= AA\&gerTypeName=\%E6\%B0\%B4\%E7\%A8\%BB\%E7\%B1\% $\mathrm{BB} \&$ isFeature $=0$ ). All the plant materials were grown in compliance with the legislation of China in the experimental paddy fields of Guangxi University according to previously described methods with minor modifications [16]. In brief, rice plants were grown in equal amounts of paddy soil. Additionally, an equal amount of water was routinely applied. The drought treatment was performed according to previously described methods [25]. In the 17-day of drought treatment, soil moisture was monitored regularly to determine the stages of drought stress using an EM-WSYP soil moisture detector (Hengmei Company, China). The penultimate leaves of 3week-old soil-cultured rice seedlings (three biological replicates) grown in the greenhouse $\left(35^{\circ} \mathrm{C}\right.$ day $/ 30^{\circ} \mathrm{C}$ night) were collected at four soil moisture points, 40 (40\% soil moisture, representing well-watered conditions), 22 (22\% soil moisture, drought treatment for $96 \mathrm{~h}$ after $40 \%$ soil moisture conditions), 20 (20\% soil moisture, drought treatment for $120 \mathrm{~h})$, and 15 (15\% soil moisture, drought treatment for $168 \mathrm{~h}$ ) (Fig. S1), and flash frozen in liquid nitrogen for RNA-seq and physiological index measurements. The samples harvested at point 15 were for physiological measurement analyses only. Drought stress treatment for hydroponically cultivated seedlings was performed in rice nutrient solution supplemented with 20\% (w/v) PEG 6000 in 96-well plates. The survival rate was determined after 5 days of treatment and rewatering for 3 days.

\section{RNA-seq library construction and transcriptomic data processing}

A transcriptome library was generated by a NEBNext ${ }^{\circ}$ Ultra $^{\text {Tu }}$ RNA Library Prep Kit for Illumina ${ }^{\circ}$ (NEB, USA), following the manufacturer's instructions. After digestion, purification, and PCR amplification, the cDNA fragments $(250 \sim 300 \mathrm{bp})$ were purified by AMPure XP beads (Beckman, USA). cDNA sequencing was performed on an Illumina 2500 platform, and paired-end reads were generated. After removing low-quality reads from the data, the read depth of $6 \mathrm{~Gb}$-clean reads that is sufficient for optimal transcriptome coverage universally were obtained and mapped to the reference genome (the Rice Annotation Project Database, https://rapdb.dna. affrc.go.jp/index.html). DEGs between each treatment point and untreated controls were detected using DESeq, with $|\log 2 \mathrm{FC}| \geq 1$ (FC, the abbreviation for fold change) and padj $\leq 0.05$ used as thresholds to identify drought- induced DEGs. Additionally, the developmentdependent DEGs were detected in the same sampling points of well-watered samples with the same criteria. Because the development-dependent DEGs could be caused by the development of rice plants, they were excluded from the final dataset.

GO enrichment analysis was performed via PlantRegMap (http://plantregmap.cbi.pku.edu.cn/). The overrepresented GO terms were visualized using ggplot2 and the GOplot R package [75]. A heatmap was generated by MEV v4.9 [76].

\section{Coexpression network analysis}

The WGCNA R package was used to construct a coexpression network and analyze the correlation of modules and physiological data according to a previously described method [47]. Raw transcriptomic datasets of all samples were filtered to remove all genes with an FPKM (Fragments Per Kilobase Million) < 1 , even for a single replicate of any sampling point of the samples. Clean data were used to generate a coexpression network. First, the soft threshold power was estimated by the pickSoftThreshold function, which provides the appropriate soft-thresholding power for network construction. Second, a correlation matrix was constructed based on the obtained soft threshold power, and afterward, a topological overlap matrix (TOM) was calculated from the transformed correlation matrix. Finally, the genes were grouped based on the topological overlap dissimilarity (1-TOM) by average hierarchical clustering using the hclust function. Gene modules were then identified using a dynamic tree cutoff algorithm (minimum cluster size of 30, merging threshold function of 0.25). Module membership (MM) was calculated based on Pearson correlations between the expression level and the module eigengenes to identify hub genes within the modules. A relatively high MM indicates that those genes have a relatively high connectivity within the module.

To correlate the physiological data with the network, module eigengenes were correlated with each physiological data point. Gene significance (GS) was used to correlate the physiological data with the expression data of individual genes.

\section{Analysis of the movement of the stomatal aperture}

Four-week-old seedlings were selected for scanning electron microscopy (SEM) analysis. The middle part of penultimate leaves was cut into $0.5-\mathrm{cm}$ pieces, which were subsequently flash frozen in liquid nitrogen to maintain stomatal morphology. An FEI Quattro S scanning electron microscope (Thermo Fisher Scientific, USA) with a freezing stage was used to examine the leaf stomatal apertures. The opening status and density of stomata were analyzed with ImageJ software. Four random fields were 
selected for counting the number of stomata in each replicate.

\section{Quantitative real-time reverse transcription PCR analysis} Total RNA was isolated from leaf samples (three biological replicates per sample). cDNA synthesis was performed by reverse transcription (RT) with a Thermo Scientific Revert Aid First Strand cDNA Synthesis Kit (Cat\# K1622) according to the manufacturer's protocol. The sequences of genes were downloaded from RAP-DB (https://rapdb.dna.affrc.go.jp/index.html). The sequences of the primers of the relevant genes were obtained from qPrimerDB (https://biodb.swu.edu.cn/qprimerdb/) (Table S1) [77]. qPCR was performed on a Roche LightCycler 480 Real-Time PCR System in $10 \mu \mathrm{L}$ reactions with a SYBR green PCR Master Mix Kit (BIO-RAD, USA), following the manufacturer's protocol. The relative expression of each gene was calculated according to the $2^{-\Delta \Delta C T}$ method [78]. The actin gene (Os11g0163100) was used as an internal reference for analysis.

\section{Determination of the relative water content of the leaves} Leaf samples were collected at each sampling point and weighed to determine the fresh weight (FW). The samples were then oven-dried to a constant dry weight (DW). The relative water content was subsequently calculated according to the following equation: RWC $(\%)=[(F W-D W) / F W] \times 100 \%$.

\section{Examination of ABA, ROS and enzyme scavenging activity} Fresh leaf samples $(0.15 \mathrm{~g})$ of PR403 and PY6 were harvested and ground in liquid nitrogen into a fine powder, after which they were resuspended in $1.35 \mathrm{~mL}$ PBS buffer $(10 \mathrm{mM}, \mathrm{pH} 7.2)$. The supernatant was used for subsequent assays after centrifugation at $5000 \mathrm{rpm}$ for 5 min. The contents and activity of $\mathrm{H}_{2} \mathrm{O}_{2}, \mathrm{SOA}, \mathrm{ABA}$, SOD, CAT, POD, and APX were measured using an enzyme-linked immunosorbent assay (ELISA) kit (MSKBIO, China) following the manufacturer's instructions.

3,3'-Diaminobenzidine (DAB) and nitro blue tetrazolium (NBT) staining were performed to detect the accumulation of $\mathrm{H}_{2} \mathrm{O}_{2}$ and SOA in the leaves following the previously described methods [16].

\section{Supplementary information}

Supplementary information accompanies this paper at https://doi.org/10. 1186/s12870-020-02705-9.

Additional file 1: Figure. S1. Diagram showing the sampling points and the corresponding phenotypes of the plant materials.

Additional file 2: Figure S2. Determination of APX activity, endogenous ABA level, and stomatal status of PR403 and PY6 under drought treatment. (A) APX activity. (B) CAT activity. (C) NBT staining of leaf samples. (D) Endogenous ABA content. (E) Leaf stomatal opening status during drought treatment. The top panel shows three levels of stomatal aperture: completely open, partially open and completely closed. The bottom panel shows the percentages of three levels of stomatal opening in PY6 and PR403 ( $n=100$ stomata for PR403 and PY6). The different letters at the top of each column in (A), (B), (D), and (E) indicate statistically significant differences based on ANOVA with Tukey's HSD test $(P<0.05)$. Scale bars $=0.5 \mathrm{~cm}$ in (B) and $=5 \mu \mathrm{m}$ in (D).

Additional file 3: Figure S3. Validation of the RNA-seq data by qRTPCR. The ACTIN gene (Os11g0163100) was used as an endogenous reference for qPCR. Os01g0164600 (A), Os01g0289600 (B), Os02g0115700 (C), Os03g0319400 (D), and Os04g0610400 (E) were selected for qPCR. The sequences of the primers used are shown in Table S1.

Additional file 4: Figure S4. Cellular component GO terms significantly overrepresented in the DEG GO enrichment analysis. (A) DEG-enriched GO terms at sampling point 22 of PR403. (B) DEG-enriched GO terms at sampling point 22 of PY6. (C) DEG-enriched GO terms at sampling point 20 of PR403. (D) DEG-enriched GO terms at sampling point 20 of PY6. Bubble size is proportional to the number of each GO-term, and the color represents the -log10 (Qvalue)

Additional file 5: Figure S5. Molecular function GO terms significantly overrepresented in the DEG GO enrichment analysis. (A) DEG-enriched GO terms at sampling point 22 of PR403. (B) DEG-enriched GO terms at sampling point 22 of PY6. (C) DEG-enriched GO terms at sampling point 20 of PR403. (D) DEG-enriched GO terms at sampling point 20 of PY6. Bubble size is proportional to the number of each GO-term, and the color represents the -log10 (Qvalue).

Additional file 6: Figure S6. Soft threshold power estimation in the WGCNA.

Additional file 7: Figure S7. Scatter plot of module eigengenes in the modules significantly correlated with $\mathrm{H}_{2} \mathrm{O} 2$ and MDA. (A) $\mathrm{H}_{2} \mathrm{O} 2$ accumulation correlated with the modules black, blue, green, and grey 60 . (B) MDA accumulation correlated with the modules red, brown, and royalblue.

Additional file 8: Figure S8. Heatmap showing that the expression patterns of the hub genes in the modules correlated with $\mathrm{H} 2 \mathrm{O} 2$ and MDA accumulation. (A) Module Black. (B) Module Blue. (C) Module Grey60. (D) Module Green. (E) Module Red. (F) Module Brown. All the data used in the analysis were subjected to $\log 2$ transformation.

Additional file 9: Figure S9. GO terms overrepresented in the GO enrichment analysis of hub genes in the modules correlated with $\mathrm{H} 2 \mathrm{O} 2$ accumulation. The $\mathrm{GO}$ terms of biological processes, cellular components, and molecular functions were overrepresented in the modules (A) black, (B) blue, (C) grey60, and (D) green, respectively. Bubble size is proportional to the number of each GO-term, and the color represents the $-\log 10$ (Qvalue).

Additional file 10: Figure S10. GO terms overrepresented in the GO enrichment analysis of hub genes in the modules correlated with MDA accumulation. The $\mathrm{GO}$ terms of biological processes, cellular components, and molecular functions were overrepresented in the modules (A) red, (B) brown, and (C) royalblue, respectively. Bubble size is proportional to the number of each GO-term, and the color represents the -log10 (Qvalue).

Additional file 11: Table S1. The primer sequences of genes using for qPCR.

Additional file 12: Table S2-1. The differentially expressed genes (DEGs) identified in the comparison of PR403_CK22vsPR403_CK40 $(\log 2 F C \geq 1$ and padj $\leq 0.05$ ). Table S2-2. The differentially expressed genes (DEGs) identified in the comparison of PR403_CK20vsPR403_CK40 ( $\log 2 F C \geq 1$ and padj $\leq 0.05$ ). Table S2-3. The differentially expressed genes (DEGs) identified in the comparison of PY6_CK22vsPY6_CK40 ( $\log 2 \mathrm{FC} \geq 1$ and padj $\leq 0.05$ ). Table S2-4. The differentially expressed genes (DEGs) identified in the comparison of PY6_CK20vsPY6_CK40 ( $\log 2 \mathrm{FC} \geq 1$ and padj $\leq 0.05$ ). Table S2-5. The development dependent DEGs identified in the comparison of PR403_CK22vsPR403_CK40 ( $\log 2 \mathrm{FC} \geq 1$ and padj $\leq 0.05$ ). Table S2-6 The development dependent DEGs identified in the comparison of PR403_CK20vsPR403_CK40 $(\log 2 \mathrm{FC} \geq 1$ and padj $\leq$ 0.05). Table S2-7. The development dependent 
DEGs identified in the comparison of PY6_CK22vsPY6 CK40 (log2FC $\geq 1$ and padj $\leq 0.05$ ). Table S2-8. The development dependent DEGs identified in the comparison of PY6 CK20vsPY6 CK40 (log2FC $\geq 1$ and padj $\leq$ 0.05). Table S2-9. The differentially expressed genes (DEGs) identified in the comparison of PR403_22vsPR403_CK40 (log2FC $\geq 1$ and padj $\leq$ 0.05). Table S2-10. The differentially expressed genes (DEGs) identified in the comparison of PR403_20vsPR403_CK40 ( $\log 2 F C \geq 1$ and padj $\leq$ 0.05). Table S2-11. The differentially expressed genes (DEGs) identified in the comparison of PY6_22vsPY6_CK40 (log2FC $\geq 1$ and padj $\leq$ 0.05). Table S2-12. The differentially expressed genes (DEGs) identified in the comparison of PY6_20vsPY6_CK40 (log2FC $\geq 1$ and padj $\leq$ 0.05).

Additional file 13: Table S3-1. Hub genes list of module black. Table S3-2. Hub genes list of module blue. Table S3-3. Hub genes list of module grey60. Table S3-4. Hub genes list of module green. Table S35. Hub genes list of module red. Table $\mathbf{5 3 - 6}$. Hub genes list of module brown. Table S3-7. Hub genes list of module royalblue.

Additional file 14: Table S4. Differentially expressed hub genes identified in module brown.

Additional file 15: Table S5-1. GO enrichment result of module black. Table S5-2. GO enrichment result of module blue. Table S5-3. GO enrichment result of module grey60. Table S5-4. GO enrichment result of module green. Table S5-5. GO enrichment result of module red. Table S5-6. GO enrichment result of module brown. Table S5-7. GO enrichment result of module royalblue. Table S5-8 GO enrichment result of PR403 22 vs PR403 CK40. Table S5-9. GO enrichment result of PR403 20 vs PR403 CK40. Table S5-10. GO enrichment result of PY6 22 vs PY6 CK40. Table S5-11. GO enrichment result of PY6 20 vs PY6 CK40.

\section{Abbreviations}

CSSL: Chromosome single-substitution segment line; DEGs: Differentially expressed genes; SNPs: Single nucleotide polymorphisms; MDA: Malondialdehyde; WGCNA: Weighted gene coexpression network analysis; ROS: Reactive oxygen species; SOD: Superoxide dismutase; CAT: Catalase; APX: Ascorbate peroxidase; TFs: Transcription factors; PR: Pathogenesis-related; POD: Peroxidase; PUFA: Polyunsaturated fatty acid PCA: Principal component analysis; BP: Biological process; CC: Cellular component; MF: Molecular function; DEHGs: Differentially expressed hub genes; TOM: Topological overlap matrix; MM: Module membership; GS: Gene significance; SEM: Scanning electron microscopy; FW: Fresh weight; DW: Dry weight; DAB: 3,3'-Diaminobenzidine; NBT: Nitro blue tetrazolium

\section{Acknowledgments}

We thank Lijun Luo, a senior researcher at Shanghai Academy of Agricultural Sciences, for providing the parental rice seeds. We thank the reviewers and editors for the careful reading of our manuscript and constructive comments, and we appreciate the technical assistance concerning the scientific instrument platform of the State Key Laboratory for Conservation and Utilization of Subtropical Agro-bioresources (Guangxi University) and are grateful for the staff performing the SEM analysis in this study.

\section{Authors' contributions}

$J L$ and $R L$ conceived and designed the experiments. BY, JBL, DW, YL, and WC performed all the lab experiments. BY, JBL, and DW conducted the field trials and collected samples for RNA-seq. JL, BY, and WC processed the data. JL drafted the manuscript. JL, RL, BY, and SW revised the manuscript. All authors approved the final version of the manuscript.

\section{Funding}

This research was supported by grants from the National Natural Science Foundation of China (CN) (31671646), the Guangxi Hundred-Talents Program (2015), the Guangxi innovation-driven development special funding project (Guike-AA17204070), and the State Key Laboratory for Conservation and Utilization of Subtropical Agro-bioresources (SKLCUSA-a201907, -a201918, and -a201801). The funding bodies had no role in the design of the study and collection, analysis, and interpretation of data and in writing the manuscript.

\section{Availability of data and materials}

All data generated or analysed during this study are included in this published article [and its supplementary information files]. The sequencing data is deposited to gene expression omnibus (GEO) database of NCBI (accession number: GSE158928; https://www.ncbi.nlm.nih.gov/geo/query/acc. cgi?acc=GSE158928).

\section{Ethics approval and consent to participate}

Not applicable.

\section{Consent for publication}

Not applicable.

\section{Competing interests}

The authors declare that they have no competing interests.

\section{Author details}

${ }^{1}$ College of Life Science and Technology (State Key Laboratory for Conservation and Utilization of Subtropical Agro-bioresources), Guangxi University, Nanning 530004, China. ${ }^{2}$ Agriculture College, Guangxi University, Nanning 530004, China. ${ }^{3}$ Agriculture College, South China Agricultural University, Guangzhou 510642, China.

Received: 11 August 2020 Accepted: 14 October 2020

Published online: 20 October 2020

\section{References}

1. de Zelicourt A, Colcombet J, Hirt H. The role of MAPK modules and ABA during abiotic stress signaling. Trends Plant Sci. 2016;21(8):677-85.

2. Verma V, Ravindran P, Kumar PP. Plant hormone-mediated regulation of stress responses. BMC Plant Biol. 2016;16:86.

3. Zhu X, Xiong L. Putative megaenzyme DWA1 plays essential roles in drought resistance by regulating stress-induced wax deposition in rice. Proc Natl Acad Sci U S A. 2013;110(44):17790-5.

4. Huber AE, Melcher PJ, Pineros MA, Setter TL, Bauerle TL. Signal coordination before, during and after stomatal closure in response to drought stress. New Phytol. 2019;224:675-88.

5. Hu H, Xiong L. Genetic engineering and breeding of drought-resistant crops. Annu Rev Plant Biol. 2014;65(1):715-41.

6. Luo LJ. Breeding for water-saving and drought-resistance rice (WDR) in China. J Exp Bot. 2010;61(13):3509-17.

7. Vahdati K, Lotfi N. Screening for drought-tolerant genotypes of Persian walnuts (Juglans regia L.) during seed germination. HortScience. 2009;44(7): 1815-9.

8. Lotfi N, Vahdati K, Amiri R, Kholdebarin B. Drought-induced accumulation of sugars and Proline in radicle and Plumule of tolerant walnut varieties during germination phase. Acta Hortic. 2010;861:289-96.

9. Lou Q, Chen L, Mei H, Wei H, Feng F, Wang P, Xia H, Li T, Luo L. Quantitative trait locus mapping of deep rooting by linkage and association analysis in rice. J Exp Bot. 2015;66(15):4749-57.

10. Lilley JM, Ludlow MM, McCouch SR, O'Toole JC. Locating QTL for osmotic adjustment and dehydration tolerance in rice. J Exp Bot. 1996:47:1427-36.

11. Huang $L$, Chen L, Wang L, Yang Y, Rao Y, Ren D, Dai L, Gao Y, Zou W, Lu X, et al. A Nck-associated protein 1 -like protein affects drought sensitivity by its involvement in leaf epidermal development and stomatal closure in rice. Plant J. 2019;98(5):884-97.

12. Huang XY, Chao DY, Gao JP, Zhu MZ, Shi M, Lin HX. A previously unknown zinc finger protein, DST, regulates drought and salt tolerance in rice via stomatal aperture control. Genes Dev. 2009;23(15):1805-17.

13. Uga Y, Sugimoto K, Ogawa S, Rane J, Ishitani M, Hara N, Kitomi Y, Inukai Y, Ono K, Kanno N, et al. Control of root system architecture by DEEPER ROOTING 1 increases rice yield under drought conditions. Nat Genet. 2013; 45(9):1097-102.

14. Aparna K, Nepolean T, Srivastsava RK, Kholová J, Rajaram V, Kumar S, Rekha $B$, Senthilvel $S$, Hash CT, Vadez V. Quantitative trait loci associated with constitutive traits control water use in pearl millet [Pennisetum glaucum (L.) R. Br.]. Plant Biol. 2015;17(5):1073-84.

15. Arab MM, Marrano A, Abdollahi-Arpanahi R, Leslie CA, Cheng H, Neale DB, Vahdati K. Combining phenotype, genotype, and environment to uncover genetic components underlying water use efficiency in Persian walnut. J Exp Bot. 2020;71(3):1107-27. 
16. Cen W, Liu J, Lu S, Jia P, Yu K, Han Y, Li R, Luo J. Comparative proteomic analysis of QTL CTS-12 derived from wild rice (Oryza rufipogon Griff.), in the regulation of cold acclimation and de-acclimation of rice (Oryza sativa L.) in response to severe chilling stress. BMC Plant Biol. 2018;18(1):163.

17. Kojima K, Oshita M, Nanjo Y, Kasai K, Tozawa Y, Hayashi H, Nishiyama Y. Oxidation of elongation factor $\mathrm{G}$ inhibits the synthesis of the D1 protein of photosystem II. Mol Microbiol. 2007;65(4):936-47.

18. Xu PL, Guo YK, Bai JG, Shang L, Wang XJ. Effects of long-term chilling on ultrastructure and antioxidant activity in leaves of two cucumber cultivars under low light. Physiol Plant. 2008;132(4):467-78.

19. Mori IC, Schroeder JI. Reactive oxygen species activation of plant Ca2+ channels. A signaling mechanism in polar growth, hormone transduction, stress signaling, and hypothetically mechanotransduction. Plant Physiol. 2004;135(2):702-8.

20. Ramachandra Reddy A, Chaitanya KV, Vivekanandan M. Drought-induced responses of photosynthesis and antioxidant metabolism in higher plants. J Plant Physiol. 2004;161(11):1189-202.

21. Wang Z, Wang F, Hong Y, Huang J, Shi H, Zhu JK. Two chloroplast proteins suppress drought resistance by affecting ROS production in guard cells. Plant Physiol. 2016;172(4):2491-503.

22. Lotfi N, Soleimani A, Vahdati K, Çakmakçı R. Comprehensive biochemical insights into the seed germination of walnut under drought stress. Sci Hortic. 2019;250:329-43.

23. Lotfi N, Vahdati K, Hassani D, Kholdebarin B, Amiri R. Peroxidase, Guaiacol peroxidase and Ascorbate peroxidase activity accumulation in leaves and roots of walnut trees in response to drought stress. Acta Hortic. 2010;861:309-16.

24. Gan P, Liu F, Li R, Wang S, Luo J. Chloroplasts- beyond energy capture and carbon fixation: tuning of photosynthesis in response to chilling stress. Int J Mol Sci. 2019;20(20):5046

25. Zong W, Tang N, Yang J, Peng L, Ma S, Xu Y, Li G, Xiong L. Feedback regulation of $A B A$ signaling and biosynthesis by a bZIP transcription factor targets drought-resistance-related genes. Plant Physiol. 2016;171(4):2810-25.

26. Liu J, Zhang C, Wei C, Liu X, Wang M, Yu F, Xie Q, Tu J. The RING finger ubiquitin E3 ligase OsHTAS enhances heat tolerance by promoting $\mathrm{H}_{2} \mathrm{O} 2$ induced Stomatal closure in Rice. Plant Physiol. 2016;170(1):429-43.

27. Bailey-Serres J, Parker JE, Ainsworth EA, Oldroyd GED, Schroeder JI. Genetic strategies for improving crop yields. Nature. 2019;575(7781):109-18.

28. Liu C, Mao B, Ou S, Wang W, Liu L, Wu Y, Chu C, Wang X. OsbZIP71, a bZIP transcription factor, confers salinity and drought tolerance in rice. Plant Mol Biol. 2014;84(1-2):19-36

29. Okamoto M, Peterson FC, Defries A, Park SY, Endo A, Nambara E, Volkman $B F$, Cutler SR. Activation of dimeric ABA receptors elicits guard cell closure ABA-regulated gene expression, and drought tolerance. Proc Natl Acad Sci U S A. 2013;110(29):12132-7.

30. Shim JS, Oh N, Chung PJ, Kim YS, Choi YD, Kim JK. Overexpression of OsNAC14 improves drought tolerance in Rice. Front Plant Sci. 2018;9:310.

31. Tang N, Ma S, Zong W, Yang N, LV Y, Yan C, Guo Z, Li J, Li X, Xiang Y, et al. MODD mediates deactivation and degradation of OsbZIP46 to negatively regulate $A B A$ signaling and drought resistance in Rice. Plant Cell. 2016;28(9): 2161-77.

32. Wang P, Zhao Y, Li Z, Hsu CC, Liu X, Fu L, Hou YJ, Du Y, Xie S, Zhang C, et al. Reciprocal regulation of the TOR kinase and ABA receptor balances plant growth and stress response. Mol Cell. 2018;69(1):100-12 e106.

33. Xiao J, Cheng H, Li X, Xiao J, Xu C, Wang S. Rice WRKY13 regulates cross talk between abiotic and biotic stress signaling pathways by selective binding to different cis-elements. Plant Physiol. 2013;163(4):1868-82.

34. Yokotani N, Sato Y, Tanabe S, Chujo T, Shimizu T, Okada K, Yamane H, Shimono M, Sugano S, Takatsuji H, et al. WRKY76 is a rice transcriptional repressor playing opposite roles in blast disease resistance and cold stress tolerance. J Exp Bot. 2013;64(16):5085-97.

35. Ross CA, Liu Y, Shen QJ. The WRKY gene family in Rice (Oryza sativa). J lintegr Plant Biol. 2007;49(6):827-42

36. Group RWW. Nomenclature report on rice WRKY's - Conflict regarding gene names and its solution. Rice (N Y). 2012;5(1):3.

37. Pandey SP, Somssich IE. The role of WRKY transcription factors in plant immunity. Plant Physiol. 2009;150(4):1648-55.

38. Takeuchi K, Gyohda A, Tominaga M, Kawakatsu M, Hatakeyama A, Ishii N, Shimaya K, Nishimura T, Riemann M, Nick P, et al. RSOsPR10 expression in response to environmental stresses is regulated antagonistically by jasmonate/ethylene and salicylic acid signaling pathways in rice roots. Plant Cell Physiol. 2011;52(9):1686-96.
39. Liu J-J, Ekramoddoullah AKM. The family 10 of plant pathogenesis-related proteins: their structure, regulation, and function in response to biotic and abiotic stresses. Physiol Mol Plant P. 2006;68(1-3):3-13.

40. Agarwal P, Agarwal PK. Pathogenesis related-10 proteins are small, structurally similar but with diverse role in stress signaling. Mol Biol Rep. 2014:41(2):599-611.

41. Varoquaux N, Cole B, Gao C, Pierroz G, Baker CR, Patel D, Madera M, Jeffers T, Hollingsworth J, Sievert J, et al. Transcriptomic analysis of field-droughted sorghum from seedling to maturity reveals biotic and metabolic responses. Proc Natl Acad Sci U S A. 2019;116(52):27124-32.

42. Zhang Z-F, Li Y-Y, Xiao B-Z. Comparative transcriptome analysis highlights the crucial roles of photosynthetic system in drought stress adaptation in upland rice. Sci Rep. 2016;6(1):19349.

43. Huang L, Zhang F, Zhang F, Wang W, Zhou Y, Fu B, Li Z. Comparative transcriptome sequencing of tolerant rice introgression line and its parents in response to drought stress. BMC Genomics. 2014;15(1):1026.

44. Kim TW, Youn JH, Park TK, Kim EJ, Park CH, Wang Z, Kim SK, Kim TW. OST1 activation by the Brassinosteroid-regulated kinase CDG1-like 1 in Stomatal closure. Plant Cell. 2018;30(8):1848-63.

45. Borah P, Sharma E, Kaur A, Chandel G, Mohapatra T, Kapoor S, Khurana JP. Analysis of drought-responsive signalling network in two contrasting rice cultivars using transcriptome-based approach. Sci Rep. 2017;7:42131.

46. Okay S, Derelli E, Unver T. Transcriptome-wide identification of bread wheat WRKY transcription factors in response to drought stress. Mol Gen Genomics. 2014;289(5):765-81.

47. Langfelder P, Horvath S. WGCNA: an R package for weighted correlation network analysis. BMC Bioinformatics. 2008;9:559.

48. Zhang B, Horvath S. A general framework for weighted gene co-expression network analysis. Stat Appl Genet Mol Biol. 2005;4(1):17.

49. Zhou XG, Huang XL, Liang SY, Tang SM, Wu SK, Huang TT, Mo ZN, Wang QY. Identifying miRNA and gene modules of colon cancer associated with pathological stage by weighted gene co-expression network analysis. Onco Targets Ther. 2018;11:2815-30.

50. Clarke C, Madden SF, Doolan P, Aherne ST, Joyce H, O'Driscoll L, Gallagher WM, Hennessy BT, Moriarty M, Crown J, et al. Correlating transcriptional networks to breast cancer survival: a large-scale coexpression analysis. Carcinogenesis. 2013;34(10):2300-8.

51. Deng T, Liang A, Liang S, Ma X, Lu X, Duan A, Pang C, Hua G, Liu S, Campanile $\mathrm{G}$, et al. Integrative analysis of Transcriptome and GWAS data to identify the hub genes associated with Milk yield trait in Buffalo. Front Genet. 2019;10:36.

52. Du J, Wang S, He C, Zhou B, Ruan YL, Shou H. Identification of regulatory networks and hub genes controlling soybean seed set and size using RNA sequencing analysis. J Exp Bot. 2017:68(8):1955-72.

53. Greenham K, Mockler TC, Weinig C, Guadagno CR, Ewers BE, Gehan MA, McClung CR. Temporal network analysis identifies early physiological and transcriptomic indicators of mild drought in Brassica rapa. eLIFE. 2017;6:e29655.

54. Shaik R, Ramakrishna W. Genes and co-expression modules common to drought and bacterial stress responses in Arabidopsis and rice. PLoS One. 2013;8(10):e77261

55. Zhang X, Feng H, Li Z, Li D, Liu S, Huang H, Li M. Application of weighted gene co-expression network analysis to identify key modules and hub genes in oral squamous cell carcinoma tumorigenesis. Onco Targets Ther. 2018;11:6001-21.

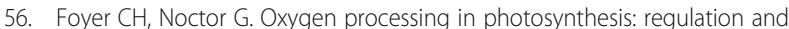
signalling. New Phytol. 2000;146(3):359-88.

57. Robinson JM, Bunce JA. Influence of drought-induced water stress on soybean and spinach leaf Ascorbate-Dehydroascorbate level and redox status. Int J Plant Sci. 2000;161(2):271-9.

58. Sah SK, Reddy KR, Li J. Abscisic acid and abiotic stress tolerance in crop plants. Front Plant Sci. 2016;7:571.

59. Ma X, Xia H, Liu Y, Wei H, Zheng X, Song C, Chen L, Liu H, Luo L. Transcriptomic and Metabolomic studies disclose key metabolism pathways contributing to well-maintained photosynthesis under the drought and the consequent drought-tolerance in Rice. Front Plant Sci. 2016;7(1886):1886.

60. Chai M, Bellizzi M, Wan C, Cui Z, Li Y, Wang G-L. The NAC transcription factor OsSWN1 regulates secondary cell wall development in Oryza sativa. J Plant Biol. 2015;58(1):44-51.

61. Davidson RM, Manosalva PM, Snelling J, Bruce M, Leung H, Leach JE. Rice Germin-like proteins: allelic diversity and relationships to early stress responses. Rice. 2010;3(1):43-55. 
62. Zhang L, Gu L, Ringler P, Smith S, Rushton PJ, Shen QJ. Three WRKY transcription factors additively repress abscisic acid and gibberellin signaling in aleurone cells. Plant Sci. 2015;236:214-22.

63. Takeuchi K, Hasegawa H, Gyohda A, Komatsu S, Okamoto T, Okada K, Terakawa T, Koshiba T. Overexpression of RSOsPR10, a root-specific rice PR10 gene, confers tolerance against drought stress in rice and drought and salt stresses in bentgrass. Plant Cell Tissue Organ Cult. 2016;127(1):35-46.

64. Li W, Zhu Z, Chern M, Yin J, Yang C, Ran L, Cheng M, He M, Wang K, Wang $J$, et al. A natural allele of a transcription factor in Rice confers broadSpectrum blast resistance. Cell. 2017;170(1):114-26 e115.

65. Zhang H, Liu Y, Wen F, Yao D, Wang L, Guo J, Ni L, Zhang A, Tan M, Jiang M. A novel rice $\mathrm{C} 2 \mathrm{H} 2$-type zinc finger protein, ZFP36, is a key player involved in abscisic acid-induced antioxidant defence and oxidative stress tolerance in rice. J Exp Bot. 2014;65(20):5795-809.

66. Choudhury FK, Rivero RM, Blumwald E, Mittler R. Reactive oxygen species, abiotic stress and stress combination. Plant J. 2017;90(5):856-67.

67. Mittler R. Oxidative stress, antioxidants and stress tolerance. Trends Plant Sci. 2002; $7(9): 405-10$

68. Mittler R. Abiotic stress, the field environment and stress combination. Trends Plant Sci. 2006;11(1):15-9.

69. Cai Z, Jia P, Zhang J, Gan P, Shao Q, Jin G, Wang L, Jin J, Yang J, Luo J. Genetic analysis and fine mapping of a qualitative trait locus wpb1 for albino panicle branches in rice. PLoS One. 2019;14(9):e0223228.

70. Li C, Ng CKY, Fan L-M. MYB transcription factors, active players in abiotic stress signaling. Environ Exp Bot. 2015;114:80-91.

71. Wang N, Zhang W, Qin M, Li S, Qiao M, Liu Z, Xiang F. Drought tolerance conferred in soybean (Glycine max. L) by GmMYB84, a novel R2R3-MYB transcription factor. Plant Cell Physiol. 2017;58(10):1764-76.

72. Naser L, Kourosh V, Bahman K, Reza A. Soluble sugars and proline accumulation play a role as effective indices for drought tolerance screening in Persian walnut (Juglans regiaL.) during germination. Fruits. 2010;65(2):97-112.

73. Bagnaresi $P$, Biselli $C$, Orrù L, Urso S, Crispino L, Abbruscato P, Piffanelli $P$, Lupotto E, Cattivelli L, Valè G. Comparative transcriptome profiling of the early response to Magnaporthe oryzae in durable resistant vs susceptible rice (Oryza sativa L.) genotypes. PLoS One. 2012;7(12):e51609.

74. Cho HY, Lee C, Hwang SG, Park YC, Lim HL, Jang CS. Overexpression of the OsChl1 gene, encoding a putative laccase precursor, increases tolerance to drought and salinity stress in transgenic Arabidopsis. Gene. 2014;552(1):98-105.

75. Walter W, Sanchez-Cabo F, Ricote M. GOplot: an R package for visually combining expression data with functional analysis. Bioinformatics. 2015; 31(17):2912-4

76. Saeed Al, Sharov V, White J, Li J, Liang W, Bhagabati N, Braisted J, Klapa M, Currier T, Thiagarajan M, et al. TM4: a free, open-source system for microarray data management and analysis. BioTechniques. 2003;34(2):374-8.

77. Lu K, Li T, He J, Chang W, Zhang R, Liu M, Yu M, Fan Y, Ma J, Sun W, et al. qPrimerDB: a thermodynamics-based gene-specific qPCR primer database for 147 organisms. Nucleic Acids Res. 2018:46(D1):D1229-36.

78. Livak KJ, Schmittgen TD. Analysis of relative gene expression data using real-time quantitative $P C R$ and the $2(-\Delta \Delta C(T))$ method. Methods. 2001; 25(4):402-8

\section{Publisher's Note}

Springer Nature remains neutral with regard to jurisdictional claims in published maps and institutional affiliations.

Ready to submit your research? Choose BMC and benefit from:

- fast, convenient online submission

- thorough peer review by experienced researchers in your field

- rapid publication on acceptance

- support for research data, including large and complex data types

- gold Open Access which fosters wider collaboration and increased citations

- maximum visibility for your research: over $100 \mathrm{M}$ website views per year

At $\mathrm{BMC}$, research is always in progress.

Learn more biomedcentral.com/submissions 